\title{
Electronically Non-Adiabatic Dynamics via Semiclassical Initial Value Methods
}

\author{
William H. Miller \\ Department of Chemistry and K. S. Pitzer Center for Theoretical Chemistry \\ University of California \\ and Chemical Sciences Division, Lawrence Berkeley National Laboratory \\ Berkeley, California 94720-1460
}

\begin{abstract}
In the late 1970's Meyer and Miller (MM) [J. Chem. Phys. 70, 3214 (1979)] presented a classical Hamiltonian corresponding to a finite set of electronic states of a molecular system (i.e., the various potential energy surfaces and their couplings), so that classical trajectory simulations could be carried out treating the nuclear and electronic degrees of freedom (DOF) in an equivalent dynamical framework (i.e., by classical mechanics), thereby describing non-adiabatic dynamics in a more unified manner. Much later Stock and Thoss (ST) [Phys. Rev. Lett. 78, 578 (1997)] showed that the MM model is actually not a 'model', but rather a 'representation' of the nuclear-electronic system; i.e., were the MMST nuclear-electronic Hamiltonian taken as a Hamiltonian operator and used in the Schrödinger equation, the exact (quantum) nuclearelectronic dynamics would be obtained. In recent years various initial value representations (IVRs) of semiclassical (SC) theory have been used with the MMST Hamiltonian to describe electronically non-adiabatic processes. Of special interest is the fact that though the classical trajectories generated by the MMST Hamiltonian (and which are the 'input' for an SC-IVR treatment) are 'Ehrenfest trajectories', when they are used within the SC-IVR framework the nuclear motion emerges from regions of non-adiabaticity on one potential energy surface (PES) or another, and not on an average PES as in the traditional Ehrenfest model. Examples are presented to illustrate and (hopefully) illuminate this behavior.
\end{abstract}




\section{Introduction}

It is well appreciated that classical molecular dynamics (MD) simulations are used nowadays to describe an enormously wide variety of chemical dynamics phenomena in quite large, complex molecular systems. In fact, it is about the only generally available theoretical tool available for carrying out such calculations. The only shortcoming of the approach is that it is, well, classical, and thus not capable of describing any quantum mechanical (QM) aspects of the dynamics that may be important; quantum effects in the dynamics of molecular systems are not always important, but sometime they are, and unless ones description is capable of providing at least an approximate description of them, one may not know whether they are important or not.

There are several ways one can proceed to try to include quantum effects in classical MD simulations; they are all approximations, of course, since only the Schrödinger equation for the complete molecular system is without approximation. Perhaps the most common class of approaches are referred to as 'mixed quantum-classical' treatments, whereby some (usually small) number degrees of freedom (DOF) are described quantum mechanically, i.e., by a wavefunction of the coordinates of these DOF that is determined via a time-dependent Schrödinger equation, and the other (typically large) number of DOF are described classically, i.e., via coordinates and momenta that follow a classical-like trajectory. The time-dependent Schrödinger equation for the quantum DOF, and classical trajectory equations for the classical DOF, are then integrated simultaneously, the coupling between the quantum and classical DOF being treated in some approximate fashion. There are a variety of such mixed quantum-classical approaches, and they have been usefully applied to a variety of problems. The primary shortcoming of such approaches is that there is no way to couple the quantum and classical DOF that is completely consistent. 
Another strategy that has been pursued recently by several groups is to use a semiclassical (SC) description for all the DOF, following approaches ${ }^{1}$ developed in the early 1970's and applied then to small molecular systems (e.g., inelastic and reactive scattering of atom-diatom collisions). ${ }^{2}$ The advantage of this SC approach is that it provides a dynamically consistent treatment of all DOF and, in fact, includes all quantum effects at least qualitatively (and usually quite quantitatively). The disadvantage of a SC treatment is that it is in general more difficult to implement than mixed quantum-classical approximations, though the resurrection $^{3}$ in recent years of various initial value representations ${ }^{1 \mathrm{a}}$ (IVRs) of SC has contributed significantly toward making SC calculations practical for large molecular systems. There have been several reviews ${ }^{4}$ of SC-IVR methodology and its applications in recent years, and I refer the reader to these for a general description of the approach and the various methods used to implement it.

Most applications of SC-IVR approaches have been to molecular dynamics on one BornOppenheimer potential energy surface (PES), and the question invariably arises as to whether or not one can use such methods to treat electronically non-adiabatic processes, i.e., those involving transitions between different PES's. The answer is 'yes', and such is the subject of this paper. Section II first reviews some relevant history and motivations of how these approaches to electronically non-adiabatic dynamics came about, and then Section III describes how modern SC-IVR implements them. Section IV discusses some of the implications.

Perhaps the most interesting aspect of the formulation described below is its relation to the classic Ehrenfest model ${ }^{5}$ for electronically non-adiabatic dynamics. It is thus useful to conclude this Introduction with a brief review and discussion of this model. It is a typical mixed quantum-classical approach, where here the electronic DOF are described QM'ly and the nuclear 
DOF treated classically. Thus if $\left\{H_{k, k^{\prime}}(\mathbf{R})\right\}, k, k^{\prime}=1, \ldots, N$ is the diabatic ${ }^{6}$ electronic matrix (as a function of the nuclear coordinates $\mathbf{R}$ ) characterizing a set of $\mathrm{N}$ electronic states and their couplings, the amplitudes $\left\{\mathrm{c}_{\mathrm{k}}(\mathrm{t})\right\}$ for being in the different electronic states at time $\mathrm{t}$ are determined by the standard time-dependent Schrödinger equation,

$$
i \hbar \dot{c}_{k}(t)=\sum_{k^{\prime}=1}^{N} H_{k, k^{\prime}}(\mathbf{R}(t)) c_{k^{\prime}}(t)
$$

and the nuclear trajectory $\mathbf{R}(\mathrm{t})$ by the classical-like equations of motion,

$$
\begin{aligned}
& \dot{\mathbf{R}}(t)=\mathbf{P}(t) / \mu \\
& \dot{\mathbf{P}}(t)=-\frac{\partial}{\partial \mathbf{R}} V_{e f f}(\mathbf{R}, t)
\end{aligned}
$$

where $\mathrm{V}_{\text {eff }}(\mathbf{R}, \mathrm{t})$ is the Ehrenfest average of the diabatic electronic matrix,

$$
V_{e f f}(\mathbf{R}, t)=\sum_{k, k^{\prime}=1}^{N} c_{k}(t)^{*} H_{k, k^{\prime}}(\mathbf{R}) c_{k^{\prime}}(t)
$$

which gives the model its name. This is also often referred to as a 'mean field' model since the effective potential is the instantaneous average over all electronic states. The initial conditions (at $t=0$, say) for these equations of motion are

$$
c_{k}(0)=\delta_{k, i}
$$

where $\mathrm{i}$ is the initial electronic state, and

$$
\mathbf{R}(0)=\mathbf{R}_{0}, \mathbf{P}(0)=\mathbf{P}_{0}
$$

where the initial conditions $\left(\mathbf{R}_{0}, \mathbf{P}_{0}\right)$ for the nuclear DOF are sampled from whatever distribution is appropriate for them.

Perhaps the most serious shortcoming of this traditional Ehrenfest model shows up in regions where the electronic coupling vanishes, e.g., in the asymptotic region after a collision; the effective potential which determines the nuclear motion is then 


$$
V_{e f f}(\mathbf{R}, t)=\sum_{k=1}^{N} H_{k, k}(\mathbf{R}) P_{k}(t)
$$

where $P_{k}(t)=\left|c_{k}(t)\right|^{2}$ is the probability of being in electronic state k, which is time-independent when there is no coupling. I.e., the nuclei end up moving on an average potential energy surface, rather than (correctly) on one potential or another depending on the electronic state of the system (i.e., the electronic and nuclear DOF are not properly correlated), and this can be grossly unphysical if the potentials for different electronic states are very different. One of the major accomplishments of the 'surface hopping' models introduced by Tully and Preston ${ }^{7}$ was to correct this unphysical feature of the Ehrenfest model.

What is remarkable, though, is that when these 'Ehrenfest trajectories', i.e., those determined by Eqs. 1.1 and 1.2, are used within SC theory, they actually emerge (correctly) on one potential surface or the other, without any 'hops' between surfaces or any other ad hoc additions to the theory. ${ }^{8}$ Showing how this comes about, and demonstrating it in a simple welldefined example, is the main purpose of this paper.

\section{Some History}

\section{a. Resonance Energy Transfer; Need for a Dynamically Consistent Model.}

The $\mathrm{F}+\mathrm{H}_{2} \rightarrow \mathrm{HF}+\mathrm{H}$ reaction was one of the most thoroughly studied chemical reactions in the 1970's, and one question regarding it was the extent to which $\mathrm{F}^{*}$, the ${ }^{2} \mathrm{P}_{1 / 2}$ excited spin-orbit state of $\mathrm{F}$, is reactive. The adiabatic PES which correlates to $\mathrm{F}^{*}$ is nonreactive, so reaction of $\mathrm{F}^{*}$ with $\mathrm{H}_{2}$ requires that there be a non-adiabatic transition to the lower (reactive) PES. Earliest considerations suggested ${ }^{9}$ that there should be little reaction of $\mathrm{F}^{*}$, since the two PES's do not experience any obvious 'avoided crossing', but later quantum scattering calculations by Lester and Rebentrost ${ }^{10}$ showed a strong electronic-to-rotational resonance 
energy transfer cross section due to the near match of the $404 \mathrm{~cm}^{-1}$ excitation energy in $\mathrm{F}^{*}$ and the $\mathrm{j}=0 \rightarrow 2$ rotational excitation energy of $\mathrm{H}_{2}\left(\sim 360 \mathrm{~cm}^{-1}\right)$. The physical picture is thus that the electronic transition to the lower, reactive PES takes place with $\mathrm{H}_{2}$ being rotationally excited, and then the reaction occurs,

$$
F^{*}+H_{2}(j=0) \rightarrow F+H_{2}(j=2) \rightarrow H F+H
$$

The near resonance of the electronic-rotational energy transfer thus enhances the non-adiabatic transition and leads to significant reaction of $\mathrm{F}^{*}$. There is a similar near electronic-to-vibrational resonance energy transfer in $\mathrm{Br}^{*}+\mathrm{H}_{2}(\mathrm{v}=0) \rightarrow \mathrm{Br}+\mathrm{H}_{2}(\mathrm{v}=1)$; i.e., when $\mathrm{Br}^{*}$ is quenched in collision with $\mathrm{H}_{2}$, essentially all the product $\mathrm{H}_{2}$ emerges in the $\mathrm{v}=1$ excited state, ${ }^{11,12}$ because the spin-orbit splitting in $\mathrm{Br}\left(3500 \mathrm{~cm}^{-1}\right)$ is not so far from the vibrational quantum of $\mathrm{H}_{2}$ $\left(\sim 4000 \mathrm{~cm}^{-1}\right)$.

However the simplest type of surface-hopping $\operatorname{model}^{7}$ (a kind of mixed-quantum classical approximation) does not describe this kind of resonance enhancement. This is easiest to illustrate ${ }^{13}$ with regard to the $\mathrm{Br}^{*}+\mathrm{H}_{2}$ example. If $\mathrm{r}$ and $\mathrm{R}$ denote the $\mathrm{H}_{2}$ vibrational and the $\mathrm{H}_{2}-$ $\mathrm{Br}$ translational coordinates, respectively (and ignoring any other coordinates in this pedagogical discussion), let $\mathrm{r}(\mathrm{t})$ and $\mathrm{R}(\mathrm{t})$ be their values along a classical trajectory, moving on BO PES $\mathrm{W}_{1}(\mathrm{r}, \mathrm{R})$, say. Within the Landau-Zener-Stuckelberg approximation ${ }^{14}$ for electronically nonadiabatic transitions, one monitors the electronic energy difference to $\mathrm{PES} \mathrm{W}_{2}$, say, as a function of time along the nuclear trajectory

$$
\Delta W(t)=W_{2}(r(t), R(t))-W_{1}(r(t), R(t)),
$$

and times at which $\Delta \mathrm{W}(\mathrm{t})$ experiences a local minimum signify 'avoided crossings' of PES's $\mathrm{W}_{1}$ and $\mathrm{W}_{2}$, and a time at which a transition between them can occur; if $\mathrm{t}_{\mathrm{n}}$ is such a time, the transition probability at this time is given approximately by ${ }^{15}$ 


$$
P_{n} \cong \exp \left[-\frac{4}{3} \Delta W_{n} \sqrt{2 \Delta W_{n} / \Delta \ddot{W}_{n}} / \hbar\right]
$$

There are in fact many such local minima in $\Delta \mathrm{W}(\mathrm{t})$ since $\Delta \mathrm{W}$ depends on the vibrational coordinate $\mathrm{r}$ (when the collision partners are close to one another), and $\mathrm{r}(\mathrm{t})$ is an oscillatory function of time,

$$
r(t) \sim \cos (\omega t)
$$

where $\omega$ is the $\mathrm{H}_{2}$ vibrational frequency. Fig. 1 shows a sketch of $\Delta \mathrm{W}(\mathrm{t})$ for two situations, indicating the various times where local minima (avoided crossings) occur. A purely classical treatment would give the net transition probability as the sum of making the transition at the various times $t_{n}$ (ignoring, for this discussion, recrossings from PES $\mathrm{W}_{2}$ back to $\mathrm{W}_{1}$ )

$$
P_{2,1}^{C L}=\sum_{n} P_{n}
$$

A SC treatment, ${ }^{15}$ a la Stuckelberg, however would first construct the net amplitude (S-matrix element) for making the transition, with the transition probability being its square modulus

$$
\begin{aligned}
& S_{2,1} \cong \sum_{n} P_{n}^{1 / 2} e^{-i \Delta W_{n} t_{n} / \hbar} \\
& P_{2,1}=\left|S_{2,1}\right|^{2}
\end{aligned}
$$

where the phase associated with each avoided crossing has been approximated as the electronic energy gap times the time of the transition. Noting that the times $t_{n}$ are spaced approximately by the period of the $\mathrm{H}_{2}$ vibration, i.e.,

$$
t_{n} \cong \text { constant }+n(2 \pi / \omega)
$$

so that

$$
S_{2,1} \cong \sum_{n} P_{n}^{1 / 2} \exp \left(-2 \pi i n \Delta W_{n} / \hbar \omega\right)
$$


If the collision were slow, so that many avoided crossings contributed with $\sim$ equal probabilities $P_{n}$ and energy gaps $\Delta W_{n}($ e.g., as depicted in Fig 1a), the sum in Eq. 2.7 would be strongly peaked when $\Delta W / \hbar \omega \cong$ an integer [recall the Fourier sum identity

$$
\left.\sum_{n=-\infty}^{\infty} e^{2 \pi i n z}=\sum_{\ell} \delta(z-\ell)\right]
$$

In more realistic situations there are not an infinite number of avoided crossing encounters (e.g., as sketched in Fig 1b), and the probability factors are not all the same, so the idealized delta function peak is broadened but still has the same qualitative effect of enhancing the transition probability near resonance (when the electronic energy gap matches the vibrational quantum). Viewed semiclassically, therefore, the enhancement of the electronic-vibrational energy transfer due to resonance energy transfer is an interference effect, ${ }^{13}$ neglecting the interference (or coherence) between the various 'hops', Eq. 2.4, misses it.

One may ask why is this the case? I.e., why is it necessary to go to a semiclassical description in order to obtain the correct resonance effect, when we know full well that resonance energy transfer is a perfectly classical phenomenon (recall the demonstrations in elementary physics class of two coupled pendulums, when their frequencies are similar)? It is because of the above mixed quantum-classical description of the system: electronic DOF are treated QM'ly, as discrete quantum states, while the vibration (and translation) DOF was treated classically, as a coordinate following a trajectory, and the inconsistency inherent in describing different DOF differently requires a SC treatment in order to describe an effect that would be described correctly classically if all the DOF were treated dynamically consistently. 


\section{b. A Classical Model for Electronic Degrees of Freedom.}

Considerations such as these led to the conclusion that if one wished to employ a classical description for the nuclear DOF — so that classical MD simulation methods could be used for them — one needed also to have a classical description of the electronic DOF. ${ }^{16-18}$ This does not necessarily mean taking the coordinates and momenta of actual electrons as classical variables - although that is appropriate in some cases, such as processes involving high Rydberg states - but rather in finding a dynamical model that characterizes the collective electronic DOF corresponding to a given finite set of electronic states.

Several heuristic ways were used to construct such classical models for a given set of electronic PES's and their couplings; for the case of two electronic states, all the approaches gave the same classical model. E.g., one of these ${ }^{16}$ exploited the fact that a two-state system is equivalent to a spin $1 / 2$ system, and then the spin was treated as a classical angular momentum. The most general approach, though, was the one of Meyer and Miller ${ }^{18}(\mathrm{MM})$, whereby the electronic amplitudes $\left\{\mathrm{c}_{\mathrm{k}}(\mathrm{t})\right\}$ in Eq. 1.1 were written in terms a pair of action-angle variables $\left\{\mathrm{n}_{\mathrm{k}}(\mathrm{t}), \mathrm{q}_{\mathrm{k}}(\mathrm{t})\right\}$ as follows

$$
c_{k}(t)=\sqrt{n_{k}(t)} e^{-i q_{k}(t)}
$$

The expectation value of the electronic Hamiltonian was then thought of a classical (timedependent) Hamiltonian of the these action-angle variables,

$$
\begin{aligned}
H_{e \ell}(\mathbf{n}, \mathbf{q} ; t) & \equiv \sum_{k, k^{\prime}=1}^{N} c_{k}(t) * H_{k, k^{\prime}}(\mathbf{R}(t)) c_{k^{\prime}}(t) \\
& =\sum_{k=1}^{N} n_{k} H_{k, k}(\mathbf{R}(t))+2 \sum_{k<k^{\prime}=1}^{N} \sqrt{n_{k} n_{k^{\prime}}} \cos \left(q_{k}-q_{k^{\prime}}\right) H_{k, k^{\prime}}(\mathbf{R}(t))
\end{aligned}
$$

where it has been assumed that $\mathrm{H}_{\mathrm{k}, \mathrm{k}}$ is a real symmetric matrix. It is not hard to show that Hamilton's equations for these (real valued) action-angle variables 


$$
\begin{aligned}
\dot{\mathbf{q}}(t) & =\frac{\partial H_{e \ell}(\mathbf{n}, \mathbf{q} ; t)}{\partial \mathbf{n}} \\
\dot{\mathbf{n}}(t) & =-\frac{\partial H_{e \ell}(\mathbf{n}, \mathbf{q} ; t)}{\partial \mathbf{q}}
\end{aligned}
$$

are identical to the time-dependent Schrödinger equation, Eq. 1.1, for the (complex valued) amplitudes. (Though we were not aware of it at the time, this classical analog for an N-state quantum system - i.e., Eqs. 2.9 - 2.11 - had been used much earlier by Dirac ${ }^{19}$ for different purposes, namely to derive the Golden Rule of time-dependent perturbation theory for treating absorption and emission of radiation.) Several ad hoc modifications were then added to Eq. 2.10: based on SC notions (i.e., the 'Langer correction') the action variables $n_{k}$ were modified as

$$
n_{k} \rightarrow n_{k}+\frac{1}{2}
$$

and then the term $1 / 2 \sum_{k} H_{k, k}$ subtracted from the Hamiltonian so that integer values of the actions would be yield one specific PES when the coupling vanished; the resulting 'classical electronic Hamiltonian' thus becomes

$$
H_{e \ell}(\mathbf{n}, \mathbf{q} ; t)=\sum_{k} n_{k} H_{k, k}(\mathbf{R}(t))+2 \sum_{k<k^{\prime}} \sqrt{\left(n_{k}+\frac{1}{2}\right)\left(n_{k^{\prime}}+\frac{1}{2}\right)} \cos \left(q_{k}-q_{k^{\prime}}\right) H_{k k^{\prime}}(\mathbf{R}(t))
$$

and adding to this the nuclear kinetic energy $\mathbf{P}^{2} / 2 \mu$ (assuming for simplicity here that all nuclear coordinates have the same mass $\mathrm{m}$ ) yields the full classical nuclear (translation, rotation, vibration)-electronic Hamiltonian for the nuclear and electronic DOF,

$$
H(\mathbf{P}, \mathbf{R}, \mathbf{n}, \mathbf{q})=\mathbf{P}^{2} / 2 \mu+H_{e \ell}(\mathbf{n}, \mathbf{q} ; \mathbf{R})
$$

The electronic action variables $\left\{\mathrm{n}_{\mathrm{k}}\right]$ play the same role, and have the same classical/semiclassical interpretation, as those for vibrational and rotational DOF. E.g., electronic state $\mathrm{k}$, say, corresponds to $\mathrm{n}_{\mathrm{k}}=1$, with the actions (SC 'quantum numbers') for all 
other electronic 'modes' being 0. It is easy to show (by using Hamilton's equations) that the sum of all the electronic actions is a constant of the motion for the Hamiltonian of Eq. 2.13,

$$
\frac{d}{d t} \sum_{k=1}^{N} n_{k}(t)=0
$$

I.e., the relevant dynamics takes place in the 'polyad' of 1 quantum of excitation in the $\mathrm{N}$ electronic DOF.

The first applications of this model Hamiltonian [Eq. 2.13] took place at the 'quasiclassical' (QC) level, ${ }^{20}$ whereby one computes classical trajectories - here for the electronic and nuclear DOF, via Hamilton's equations — with quantized initial conditions for the bounded DOF, i.e., the rotational, vibrational, and now also electronic DOF. This prescription chooses the initial action variables of the bounded DOF as integers, corresponding to the chosen initial state, and the conjugate angle variables as random [in the interval $(0,2 \pi)$ ], and the final values of the action variables are 'binned' (or histogrammed) into quantum number bins to determine the distribution of final quantum states. As with rotation and vibration, where the initial conditions are specified in action-angle variables but transformed ${ }^{21}$ into Cartesian coordinates and momenta for the actual numerical trajectory calculation, this was also done for the electronic DOF. The Cartesian electronic-oscillator variables are defined in the usual way in terms of their action-angle variables,

$$
\begin{aligned}
& x_{k}=\sqrt{2 n_{k}+1} \cos q_{k} \\
& p_{k}=-\sqrt{2 n_{k}+1} \sin q_{k}
\end{aligned}
$$

and in terms of them the nuclear-electronic Hamiltonian of Eq. 2.13 becomes

$$
H(\mathbf{P}, \mathbf{R}, \mathbf{p}, \mathbf{x})=\mathbf{P}^{2} / 2 \mu+\sum_{k=1}^{N} \frac{1}{2}\left(p_{k}^{2}+x_{k}^{2}-1\right) H_{k, k}(R)+\sum_{k<k^{\prime}=1}^{N}\left(p_{k} p_{k^{\prime}}+x_{k} x_{k^{\prime}}\right) H_{k k^{\prime}}(R)
$$


It is of course quite a 'stretch' for the quasi-classical model to treat the electronic DOF this way, for the electronic 'quantum numbers' $n_{k}$ can never be anything other than 0 or 1 (since their sum $=1$ ), and it is well known that the QC treatment for vibration and rotation, say, works best when many quantum states are populated by the dynamics and their quantum numbers are $>>0$. Nevertheless, a variety of applications were carried out this way in the late 1970's and gave quite reasonable results. E.g., Figure 2 shows a comparison ${ }^{22}$ of the $3 \mathrm{~d}$ non-reactive quenching of $\mathrm{F}^{*}$ by collision with $\mathrm{H}_{2}(\mathrm{j}=0)$, demonstrating the resonance effect discussed above; i.e., most of the product has $\mathrm{H}_{2}$ excited to the $\mathrm{j}=2$ state. And the results are in reasonable agreement with Lester and Rebentrost's ${ }^{10}$ coupled-channel quantum scattering calculations. Fig. 3 shows a similar comparison $^{23}$ for $\mathrm{Br}^{*}+\mathrm{H}_{2}(\mathrm{v}=0)$, where the cross for forming $\mathrm{H}_{2}$ in the $\mathrm{v}=1$ state is much larger than that for $\mathrm{v}=0$. Fig. 4 shows the cross section for charge transfer, $\mathrm{Na}+\mathrm{I} \rightarrow \mathrm{Na}^{+}+\mathrm{I}^{-}$, a classic ionic-covalent curve-crossing problem, ${ }^{24}$ again with quite reasonable agreement with exact QM calculations. ${ }^{25}$ Probably one feature that makes the QC version of this nuclearelectronic model work as well as it does, is that the electronic DOF are essentially harmonic oscillators, as is clear from the form of the Hamiltonian Eq. 2.16, and it is well known that classical and semiclassical descriptions often work fortuitously well for harmonic systems even when the quantum numbers (actions variables) are small. Still, one cannot always expect this QC version of the model to work as well as the examples noted here.

One possible way to improve matters is to implement the model semiclassically, i.e., within the framework of 'classical S-matrix' theory. ${ }^{\text {la }}$ Here one finds specific classical trajectories (of the full nuclear-electronic system as above) that begin and end with integer values of the action variables of all bounded DOF, e.g., vibrational, rotational, and electronic, of the nuclear-electronic Hamiltonian, and can express the S-matrix (the matrix of transition 
amplitudes) in terms of them. This was done for a model problem in the original $\mathrm{MM}^{18}$ paper, and was indeed seen to be much more accurate than the QC implementation. The difficulties of applying the classical S-matrix approach to systems with many DOF, however, prevented further applications with it. The next Section, however, discusses more recent advances using IVR methods to implement the SC approximation.

There are two final points worth pointing out before concluding this lengthy historical review. First, it is possible to use an adiabatic representation rather than a diabatic representation as has been done so far. This is a straight-forward classical canonical transformation and was carried out in the Appendix of the MM paper. Using Cartesian electronic variables, as in Eq. 2.16 (though here they are 'new' electronic variables, for which a different notation is not used since it is unnecessary for present purposes), the adiabatic nuclear-electronic Hamiltonian is

$$
H(\mathbf{P}, \mathbf{R}, \mathbf{p}, \mathbf{x})=\frac{1}{2}|\mathbf{P}+\Delta \mathbf{P}|^{2}+\sum_{k=1}^{N} \frac{1}{2}\left(p_{k}^{2}+x_{k}^{2}-1\right) E_{k}(\mathbf{R})
$$

where $\left\{\mathrm{E}_{\mathrm{k}}(\mathbf{R})\right\}$ are the Born-Oppenheimer PES's (i.e., the eigenvalues of the diabatic electronic matrix $\left.\left\{\mathrm{H}_{\mathrm{k}, \mathrm{k}},(\mathbf{R})\right\}\right), \mid \psi_{\mathrm{k}}>$ denote the $\mathrm{BO}$ electronic wavefunctions (the eigenvectors of the diabatic electronic matrix), and $\Delta \mathbf{P}$ is given by

$$
\Delta \mathbf{P}=\sum_{k<k^{\prime}=1}^{N} \hbar\left(p_{k^{\prime}} x_{k}-p_{k} x_{k^{\prime}}\right)<\psi_{k} \mid \frac{\partial \psi_{k^{\prime}}}{\partial \mathbf{R}}>
$$

One notes that this classical nuclear-electronic Hamiltonian has the same form as the QM version, involving the same non-adiabatic coupling elements which characterize the quantum non-adiabatic dynamics.

Finally, it is important to emphasize that the classical trajectories which result from this classical nuclear-electronic Hamiltonian [Eq. 2.16] are essentially identical to the Ehrenfest equations, Eqs. 1.1 and 1.2 above. (This is easy to verify by writing out Hamilton's equations 
from Eq. 2.16, and noting the expression for the electronic amplitudes in terms of the Cartesian electronic variables, $\left.c_{k}=\left(x_{k}+i * p_{k}\right) / \sqrt{2}\right)$. I say 'essentially' equivalent because there is a slight difference due to the modification made in Eq. 2.12, which introduces a zero point energy (ZPE) into the electronic DOF; without this ZPE the trajectories would not depend on the initial values the electronic angle variables, so for a given electronic state (and given initial conditions of the nuclear DOF) there would be only one trajectory. With this modification, there is an ensemble of trajectories for a given electronic state, so that even at the primitive quasi-classical level of binning the final electronic actions, one has different nuclear trajectories for different final electronic states (unlike the traditional Ehrenfest model which has the same nuclear trajectory for all final electronic states).

\section{Implementation via the Initial Value Representation of SC Theory}

\section{a. Stock and Thoss}

The most important next step in this story is the paper of Stock and Thoss ${ }^{26}(\mathrm{ST})$, whose goal was to develop a continuous representation of the electronic DOF in order to be able to apply an initial value representation (IVR) of semiclassical theory (specifically the Herman-

Kluk, coherent state IVR; ${ }^{3 a, b}$ see below). The approach they used was a procedure due to Schwinger for mapping a finite set of quantum states onto a set of bosons (i.e., harmonic oscillators), and the nuclear-electronic Hamiltonian they obtained was identical to the MM result in Eq. 2.16. What is important about the ST result is that it shows this Hamiltonian not to be a model, i.e., approximation, but rather an exact representation of the nuclear-electronic system. I.e., were one to take this as the Hamiltonian operator in a Schrödinger equation, the exact quantum dynamics would result. 
Perhaps the easiest way to demonstrate this is simply to compute the electronic matrix of the Hamiltonian operator corresponding to the classical Hamiltonian of Eq. 2.16, using the $\mathrm{N}$ electronic' oscillator basis functions $\left\{\Phi_{k}(\mathbf{x})\right\}$; these are a product of $\mathrm{N}$ harmonic oscillator eigenfunctions that have one quantum of excitation in mode $\mathrm{k}$ and none in all the others,

$$
\begin{aligned}
\Phi_{k}(\mathbf{x}) & =\phi_{1}\left(x_{k}\right) \prod_{\substack{k^{\prime} \neq 1 \\
k^{\prime} \neq k}}^{N} \phi_{0}\left(x_{k^{\prime}}\right) \\
& =\sqrt{2} \pi^{-N / 4} x_{k} e^{-\frac{1}{2} \mathbf{x} \cdot \mathbf{x}}
\end{aligned}
$$

It is a simple matter to compute the matrix elements $\left\langle\Phi_{k}|\hat{H}| \Phi_{k^{\prime}}>\right.$ (since they are elementary harmonic oscillator matrix elements); the nuclear kinetic energy term in the Hamiltonian is diagonal, proportional to the unit matrix $\delta_{k, k^{\prime}}$, and the result for the remainder is $H_{k, k^{\prime}}(R)$, the original diabatic electronic matrix. Since the matrix of the electronic-nuclear Hamiltonian is the same as that with the original diabatic electronic basis, the resulting quantum mechanics must therefore be the same.

The only approximation involved in using what I will now call the MMST Hamiltonian, Eq. 2.16, is therefore the dynamical method used to implement it - e.g., the quasi-classical model discussed above, or the SC-IVR methodology discussed below—and not the fundamental Hamiltonian itself. This Hamiltonian — within the (conserved) polyad of one quantum of excitation in the 'electronic oscillators' - is completely equivalent to the original quantum mechanical nuclear-electronic system.

\section{b. The Initial Value Representation}

Initial value representations (IVRs) were introduced ${ }^{\text {la }}$ very early in semiclassical theory (as a way to deal with 'classically forbidden' processes), and they have re-emerged ${ }^{3,4}$ in recent 
years as a practical way for dealing with molecular systems with many degrees of freedom.

They replace the non-linear boundary value problem of 'pure' semiclassical approaches, such as 'classical S-matrix' theory, by a Monte Carlo average over the initial values of classical trajectories, and this is more amenable for systems with many degrees of freedom for it allows one to utilize much of the Monte Carlo methodology that has been developed for classical MD simulations. SC-IVR methods and their applications have been reviewed several times in recent years, so here I will only summarize the basic ideas that are necessary for present applications.

One can state the SC-IVR succinctly as an expression for the propagator (time evolution operator), $\exp (-i \hat{H} t / \hbar)$. The Herman-Kluk, ${ }^{3 \mathrm{a}, \mathrm{b}}$ or coherent state version is

$$
e^{-i \hat{H} t / \hbar}=(2 \pi \hbar)^{-F} \int d \mathbf{p}_{0} \int d \mathbf{q}_{0} C_{t}\left(\mathbf{p}_{0}, \mathbf{q}_{0}\right) e^{i S_{t}\left(\mathbf{p}_{0}, \mathbf{q}_{0}\right) / \hbar}\left|\mathbf{p}_{t}, \mathbf{q}_{t}><\mathbf{p}_{0}, \mathbf{q}_{0}\right|
$$

where $\left(\mathbf{q}_{0}, \mathbf{p}_{0}\right)$ are the initial conditions of the coordinates and momenta for classical trajectories, and $\left(\mathbf{q}_{t}, \mathbf{p}_{t}\right)$ are their values at time $t$ later; $S_{t}$ is the classical action integral (the time integral of the Lagrangian) along this trajectory, and the pre-exponential factor $\mathrm{C}_{\mathrm{t}}$ involves the monodromy matrix (the matrix of derivations of $\mathbf{q}_{\mathrm{t}}$ and $\mathbf{p}_{\mathrm{t}}$ with respect to $\mathbf{q}_{0}$ and $\mathbf{p}_{0}$ ). The coherent states $\mid \mathbf{p}, \mathbf{q}>$ in Eq. 3.2 are standard Cartesian minimum uncertainty wavepackets,

$$
\left\langle\mathbf{q}^{\prime}\right| \mathbf{p}, \mathbf{q}>=\left(\frac{|\gamma|}{\pi}\right)^{F / 4} \exp \left[-\frac{1}{2}\left(\mathbf{q}^{\prime}-\mathbf{q}\right) \cdot \gamma \cdot\left(\mathbf{q}^{\prime}-\mathbf{q}\right)+i \mathbf{p} \cdot\left(\mathbf{q}^{\prime}-\mathbf{q}\right) / \hbar\right]
$$

where $\mathrm{F}$ is the number of DOF of the system, and the matrix $\gamma$ can be any positive matrix chosen for convenience. The simpler coordinate space IVR has the same form as Eq. 3.2, with the coherent states replaced by Dirac position eigenstates,

$$
\begin{aligned}
& \left|\mathbf{p}_{0}, \mathbf{q}_{0}\right\rangle \rightarrow\left|\mathbf{q}_{0}\right\rangle \\
& \left|\mathbf{p}_{t}, \mathbf{q}_{t}\right\rangle \rightarrow\left|\mathbf{q}_{t}\right\rangle
\end{aligned}
$$


and with a different pre-exponential factor (but still involving elements of the monodromy matrix); it can be obtained from Eqs. 3.2, 3.3 by taking the limit $\gamma \rightarrow \infty$.

Since the primary interest in SC-IVR methods is to use them as a way to add quantum effects to classical MD simulations of large molecular systems, their formulation has followed classical MD methodology as closely as possible. Thus Cartesian coordinates are used essentially exclusively, eschewing more sophisticated SC methods such as action-angle variables, Airy function uniformization, etc., that are useful for analytical analysis and treatments, but which do not lend themselves to generic computational implementation. Exceptions to this 'total Cartesian' approach are that sometimes covalent bond distances are fixed and only low frequency, large amplitude motions treated; this is common in classical MD simulations and has also been used analogously in SC-IVR approaches. ${ }^{27,28}$

Much of the effort in the SC-IVR arena has been their use to evaluate various time correlation functions of the form

$$
C_{A B}(t)=\operatorname{tr}\left[\hat{A} e^{i \hat{H} t / \hbar} \hat{B} e^{-i \hat{H} t / \hbar}\right]
$$

since most quantities of interest in the dynamics of complex systems can be expressed in terms of them. Straight-forward use of Eq. 3.2 for the two propagators in the correlation function thus gives it as a double phase space average

$$
\begin{aligned}
C_{A B}(t)= & (2 \pi \hbar)^{-F} \int d \mathbf{p}_{0} \int d \mathbf{q}_{0}(2 \pi \hbar)^{-F} \int d \mathbf{p}_{0}{ }^{\prime} \int d \mathbf{q}_{0}{ }^{\prime} C_{t}\left(\mathbf{p}_{0}, \mathbf{q}_{0}\right) C_{t}\left(\mathbf{p}_{0}{ }^{\prime}, \mathbf{q}_{0}{ }^{\prime}\right) * \\
& e^{i\left[S_{t}\left(\mathbf{p}_{0}, \mathbf{q}_{0}\right)-S_{t}\left(\mathbf{p}_{0}{ }^{\prime}, \mathbf{q}_{0}{ }^{\prime}\right)\right] / \hbar}<\mathbf{p}_{0}, \mathbf{q}_{0}|\hat{A}| \mathbf{p}_{0}{ }^{\prime}, \mathbf{q}_{0}{ }^{\prime}><\mathbf{p}_{t}{ }^{\prime}, \mathbf{q}_{t}{ }^{\prime}|\hat{B}| \mathbf{p}_{t}, \mathbf{q}_{t}>
\end{aligned}
$$

and here one sees the additional difficulty of an SC calculation compared to a purely classical one: since these phase space averages will of necessity be evaluated by Monte Carlo methods (both classically and SC'ly), the oscillatory factor arising from the phase difference from the action integrals of the two trajectory makes straight-forward Monto Carlo methods very 
inefficient. There are several ways that have been developed for dealing with this $\mathrm{SC}$ version of the 'sign problem'.

The most drastic approximation to Eq. 3.6 is to assume that the only parts of the integrand that contribute significantly are when the two phase points, $\left(\mathbf{p}_{0}, \mathbf{q}_{0}\right)$ and $\left(\mathbf{p}_{0}^{\prime}, \mathbf{q}_{0}{ }^{\prime}\right)$ - and thus the two trajectories emanating from them — are infinitesimally close together. Carrying through this idea (one needs to use the coordinate IVR, cf. Eq. 3.4, etc., rather than the coherent state version) by linearizing the difference between the two trajectories, ${ }^{29}$ leads to the classical Wigner approximation for the correlation function,

$$
C_{A B}(t)=(2 \pi \hbar)^{-F} \int d \mathbf{p}_{0} \int d \mathbf{q}_{0} A_{w}\left(\mathbf{p}_{0}, \mathbf{q}_{0}\right) B_{w}\left(\mathbf{p}_{t}, \mathbf{q}_{t}\right)
$$

i.e., the same expression as the classical correlation function except that the Wigner functions for the two operators appear rather than the classical functions themselves,

$$
O_{w}(\mathbf{p}, \mathbf{q})=\int d \Delta \mathbf{q} e^{-i \mathbf{p} \cdot \Delta \mathbf{q} / \hbar}<\mathbf{q}+\Delta \mathbf{q} / 2|\hat{O}| \mathbf{q}-\Delta \mathbf{q} / 2>
$$

for any operator $\hat{O}$. The classical Wigner model is an old idea, and is obtained as an approximation to a variety of formulations. ${ }^{30}$ It is interesting in the present context to see that it is contained within the SC-IVR description, and thus any more accurate treatment of the SC-IVR approach will presumably be even more accurate. The classical Wigner model cannot describe any true quantum coherence features - because the forward and backward trajectories ${ }^{31}$ are assumed to be infinitesimally close — but it does describe some quantum effects to a useful level of accuracy. It is has been shown to describe tunneling ${ }^{32}$ in $\mathrm{H}$ atom barrier transmission, for example, to an accuracy of $\sim 10-30 \%$ when the tunneling correction is as large as $10^{2}$ to $10^{3}$. It also has been seen to describe zero point energy effects ${ }^{33}$ in low temperature liquid parahydrogen which prevent the liquid from freezing (as it does classically). Because the classical Wigner model is so similar to a classical calculation, and thus relatively easy to implement, there 
has been a great deal of activity in recent years using it to calculate a variety of time correlation functions for large molecular systems.

The forward-backward (FB) IVR ${ }^{34}$ is the simplest version of the SC-IVR that goes beyond the linearized IVR/classical Wigner model and is thus able to describe true coherence effects. The basic idea ${ }^{35}$ of the FB-IVR is to combine the forward and backward propagators, $\exp (-i \hat{H} t / \hbar)$ and $\exp (+i \hat{H} t / \hbar)$, into one forward-backward propagation and thus cancel out analytically as much as possible of the phase difference between the forward and backward propagators, rather than having to do it all numerically. E.g., if operator $\hat{B}$ involved only the coordinate of one DOF, $\mathrm{q}_{1}$ say, and this were separable from all the other DOF, then the forward and backward propagators for the 'bath' (all the DOF except \# 1) would exactly cancel each other. The idea of the FB-IVR is not to make this approximation, but nevertheless obtain efficiency in the Monte Carlo integration by exploiting the fact that there will in general be much cancellation between the forward and backward propagators.

For example, if operator $\hat{B}$ is a coordinate operator that is the function of one collective function of all the coordinates $\mathbf{q}-$ i.e., $\hat{B}=B(s(\mathbf{q}))$ - as is often the case, then the FB-IVR expression for the correlation function is ${ }^{34}$

$$
C_{A B}(t)=\int d p_{s} \tilde{B}\left(p_{s}\right)(2 \pi \hbar)^{-F} \int d \mathbf{p}_{0} \int d \mathbf{q}_{0}<\mathbf{p}_{0}{ }^{\prime}, \mathbf{q}_{0}{ }^{\prime}|\hat{A}| \mathbf{p}_{0}, \mathbf{q}_{0}>e^{i S_{0}\left(\mathbf{p}_{0}, \mathbf{q}_{0}\right) / \hbar} C_{0}\left(\mathbf{p}_{0}, \mathbf{q}_{0}\right)
$$

where $\left(\mathbf{p}_{0}, \mathbf{q}_{0}\right)$ are the initial conditions for a trajectory that is propagated by the usual classical equations of motion until time $\mathrm{t}$, at which time it undergoes a momentum jump,

$$
\mathbf{p}_{t} \rightarrow \mathbf{p}_{t}+p_{s} \frac{\partial s\left(\mathbf{q}_{t}\right)}{\partial \mathbf{q}_{t}}
$$

and is then propagated back to time 0 , the final phase point being $\left(\mathbf{p}_{0}{ }^{\prime}, \mathbf{q}_{0}{ }^{\prime}\right)$. The pre-exponential factor for this forward-backward trajectory is of the usual form, and the FB action integral is the 
sum of that for the forward and backward parts of the FB trajectory plus a contribution from the

momentum jump. $\tilde{B}\left(p_{s}\right)$ is the (1d) Fourier transform of $B(\mathrm{~s})$. The correlation function is thus seen to be a single phase space average over initial conditions — not the double phase space average of the complete SC-IVR expression, Eq. 3.6 - albeit over FB trajectories, plus the 1d integral over the momentum jump parameter $p_{\mathrm{s}}$. This is essentially as simple a result as one can obtain within the SC-IVR framework that is able to describe true quantum coherence, and applications to several interesting problems have shown it to be capable of describing quantum coherence quite well. If one were to assume that only small values of $p_{\mathrm{s}}$ were significant, and expanded the FB action integral (and other factors) in Eq. 3.8 to first order in $p_{\mathrm{s}}$, then one would obtain a result very similar to the classical Wigner model (with Husimi distribution functions rather than Wigner ones).

\section{c. SC-IVR for the MMST Hamiltonian}

Application of the SC-IVR to the MMST nuclear-electronic Hamiltonian, Eq. 2.16, to treat electronically non-adiabatic processes is now straight-forward: ${ }^{36}$ classical trajectories are computed from this classical Hamiltonian, for the nuclear and electronic DOF, and the calculations can be carried out at the full SC-IVR without further approximation, e.g., Eq. 3.6, or with additional approximations, such as the 'linearized' IVR/classical Wigner approximation of Eq. 3.7, or the more accurate FB-IVR approach of Eq. 3.8. A number of such calculations have been carried out, ${ }^{37}$ using all of these approaches, and some involving many $(>>10)$ DOF and usually 2 (and some 3) electronic states, and they have generally shown very good agreement with more complete quantum calculations where these have been available. Also, Stock and 
Thoss ${ }^{38}$ have recently published a comprehensive review of the SC-IVR/MMST approach, and Grossmann ${ }^{39}$ even more recently a briefer one.

It is thus not my purpose here to survey all recent applications of the SC-IVR/MMST approach, but rather I will focus on an application ${ }^{8}$ to a very simple system, one nuclear DOF (translation, i.e., a 1d scattering problem) and two electronic states, very much like the examples that Tully ${ }^{40}$ used several years ago to test the 'fewest switches' version of his 'surface hopping' model. Fig. 5 shows the PES's (here potential curves for just this one nuclear DOF), both diabatic and adiabatic, for a symmetric and asymmetric version of the model. One imagines the incident particle approaching from the left $(\mathrm{R}<<0)$ with a well-defined translational energy on one of the potential curves, and having some probability of emerging to the 'product' region on the right (transmission) on either potential curve (i.e., electronic state), or back to the 'reactant' region on the left (reflection) also on either of the two potential curves.

The point of this calculation is to demonstrate how the MMST classical Hamiltonian behaves when it is implemented semiclassically. As has been emphasized (cf. end of Sec II), the nuclear-electronic trajectories generated by this Hamiltonian are Ehrenfest trajectories, and we know that if the traditional Ehrenfest model were applied to this problem the nuclear trajectory would emerge moving, incorrectly, on an average potential energy curve. How is this changed if these same trajectories are used within a SC description? And how 'rigorous' must be the SC treatment in order for the situation to be described correctly?

To answer these questions we calculate the probability distribution of the final translational momentum, which can be expressed as the long time limit of a correlation function like that in Eq. 3.5,

$$
P\left(P_{f}\right)=\lim _{t \rightarrow \infty} \operatorname{tr}\left[\hat{A} e^{i \hat{H} t / \hbar)} \delta\left(\hat{P}-P_{f}\right) e^{-i \hat{H} t / \hbar t}\right]
$$


where operator $\hat{A}$ is the projection operator for the initial state

$$
\hat{A}=\left|\Psi_{i}><\Psi_{i}\right|
$$

the wavefunction for which is

$$
\Psi_{i}\left(x_{1}, x_{2}, R\right)=\phi_{0}\left(x_{1}\right) \phi_{1}\left(x_{2}\right)<R \mid P_{i} R_{i}>
$$

the nuclear wavefunction being a coherent state with $\mathrm{R}_{\mathrm{i}}<<0$, and $\mathrm{P}_{\mathrm{i}}$ the initial translational momentum. (The coherent state parameter $\gamma$ for this coherent state is chosen to be quite small, so that the initial translational energy is very sharply defined.) With one quantum of excitation in 'electronic oscillator' 2, this wavefunction corresponds to the system being initially on potential curve 2. Operator $\hat{B}$ in Eq. 3.9a is a delta function, where $\hat{P}$ is the nuclear momentum operator and $\mathrm{P}_{\mathrm{f}}$ the observed final value of the nuclear (here, translational) momentum. Note that nothing in this quantity, Eq. 3.9a, refers to the final electronic state; it is a 'measurement' (i.e., observation) only of the final nuclear momentum. The reader should see ref. 8 for details of the calculations (e.g., since here operator $B$ is a function of the nuclear momentum operator, in the FB trajectories there is a jump in the nuclear position at time $t$, rather than a jump in momentum as in $\mathrm{Eq} 3.8 \mathrm{~b}$ that arises when $B$ is a function only of coordinates).

Figures 6 and 7 show the probability distribution in final translational momentum for the symmetrical potential (Fig 5a) for two different values of the initial translational energy, Fig 6 for the higher energy, and Fig 7 for the lower. (The two energies are indicated by arrows on the vertical axis in Fig 5.) Figures 6 a and 7 a show the results given by the FB version of the SCIVR, along with the exact QM results, the two being seen to be in good agreement. Since the initial state has a well-defined value for the total energy (electronic plus translational), the two peaks in the distribution for positive momentum obviously correspond to the two possible final electronic states in which the system can emerge in the 'product' (transmission) region; there is 
essentially no reflection to the 'reactant' (negative momentum) region for this potential. The areas under the peaks give the transition probabilities to each final electronic state. Figures $6 \mathrm{~b}$ and $7 \mathrm{~b}$ show the corresponding results given by the traditional Ehrenfest and the classical Wigner models. The Ehrenfest result is essentially a delta function at one particular momentum, since it involves only one trajectory; this is a manifestation of the defect of this model, namely that the nuclear trajectory emerges on an average PES, thus having only one final nuclear momentum. The classical Wigner model, since it involves an ensemble of trajectories, is somewhat better, showing a distribution of final nuclear momenta, and one sees that the breadth of the distribution is approximately related to the two peaks in Figs 6a and 7a. It cannot, though, properly describe the two peaks in the distribution which correspond to the two individual final electronic states.

Figures 8 and 9 show similar results for the asymmetric version of the model (Fig $5 \mathrm{~b}$, the two arrows on the vertical axis again indicating the two energies considered). For the higher energy, the correct QM and the FB-IVR results in Fig 8a (in good agreement with one another) show two peaks for positive momentum, corresponding again to the two final electronic states in the 'product' (transmission) region; there is essentially no reflection at this energy. The traditional Ehrenfest and classical Wiger results are shown in Fig 8b; the Ehrenfest result again shows only a single peak, as it must, at an average final nuclear momentum, while the Wigner model gives a distribution that is a crude approximation to the correct (quantized) distribution of the translational momentum. The lower energy case, Figure 9, is even more interesting because at this energy only one electronic state is allowed (an 'open channel') in transmission, while both states are allowed in reflection. Fig 9a shows the correct QM and the FB-IVR results, which are reasonable agreement (though the Fourier transform involved in the FB calculation is somewhat 
noisy); there is one peak in transmission, corresponding to the one possible electronic state, and two peaks at negative momentum (reflection) corresponding to the two possible final electronic states in the 'reactant' region. The corresponding Ehrenfest and classical Wigner results are shown in Fig 9b. Here the Ehrenfest model is almost completely useless, since one trajectory can clearly not describe transmission and reflection even qualitatively correctly; thus the one peak it shows in Fig 9b is essentially meaningless. Again, the Wigner model is better, showing some transmission and some reflection, though again it does not describe the two individual electronic states in the transmission (negative momentum) region.

\section{Discussion}

One sees, therefore, that when 'Ehrenfest trajectories' are used within a SC theory that is capable of describing interference/coherence correctly — as, for example, the FB version of the SC-IVR — the nuclear trajectories emerge, correctly, on one PES or the other, not on an average PES as in the traditional Ehrenfest model or the 'linearized'/classical Wigner approximation to the SC-IVR, which is unable to describe coherence. I.e. it is the proper inclusion of coherence that causes the Ehrenfest trajectories to 'quantize' on one PES or another; there do exist Ehrenfest trajectories that emerge on an average PES, but the net amplitude associated with them is zero because of destructive interference. This is easily understood from simple WKB theory, where bounded motion for an extended time (i.e., many periods of oscillation) in any $1 \mathrm{~d}$ potential well leads to quantization, i.e., an integer value of the classical action variable (the SC 'quantum number') as the SC phase $\exp (i S / \hbar)$ accumulates, as it does for these1d harmonic oscillators in the asymptotic region. Since there is only one quantum of excitation in the $\mathrm{N}$ electronic oscillators, and this is conserved by the dynamics, the only possible integer values for these SC 
'quantum numbers' are 1 for one of the oscillators and 0 for all others, so that the nuclear motion must emerge from an uncoupled region (after a number of vibrational periods of the electronic oscillators) on one or another of the N PES's (diabatic or adiabatic, the result being invariant to which representation is used).

It is interesting to contrast this description to that in several phenomenological models ${ }^{41,42}$ of electronically non-adiabatic dynamics in which it is $d e$-coherence that drives the nuclear trajectory to one PES or another. It would be interesting to see how these seemingly difference perspectives might be reconciled.

There are of course cases where the correct description of coherence is not crucial. The resonance electronic-vibration/rotation energy transfer effects discussed in Sec II were described reasonably well by the 'quasi-classical' treatment of the MMST Hamiltonian. The 'linearzied' IVR/classical Wigner model is very similar to the quasi-classical approximation; as seen in the example discussed in Sec IIIc, it gives a distribution of final translational momenta, which could be 'binned' to obtain transition probabilities to individual final electronic states, even though the nuclear trajectories to not emerge precisely on the corresponding final PES.

Seeing how a sufficiently rigorous implementation of SC theory is able to give a proper treatment of nuclear-electronic dynamics within the MMST representation of the system, one can also explore additional approximations that could be introduced to make calculations simpler while still retaining as correct a description as possible. For the dynamics to 'quantize' correctly on one PES or another in non-interacting regions, at least the coherence (i.e., the SC phase) of the electronic-oscillator DOF needs to be included, but perhaps one could ignore the coherence aspects of the nuclear DOF, i.e., using a more classical-like description of them. One way to implement this idea is to make a 'linearized'/classical Wigner-like approximation for the nuclear 
DOF, while still retaining a full SC description of the electronic DOF. This type of 'mixed classical/semiclassical' model has been formulated ${ }^{43}$ and model calculations carried out to illustrate its possibilities, but further applications have not been pursued. In light of the present MMST representation for nuclear-electronic dynamics, it may be worthwhile to re-visit this approximation.

Finally, it is perhaps useful to comment briefly on the relation of the SC-IVR treatment of the MMST Hamiltonian to a much earlier SC approach to electronically non-adiabatic dynamics by Pechukas, ${ }^{44}$ which was very insightful and influential; see ref. 36 for a more detailed discussion. Pechukas' approach is a two-step procedure, first to solve the (time-dependent) electronic Schrödinger equation for a given nuclear path $\mathbf{R}(\mathrm{t})$, and secondly to perform a Feynman path integral (that would give exact QM results if done exactly) over nuclear paths by the stationary phase approximation, i.e., SC'ly. This leads to a nuclear trajectory that does indeed begin and end on a specific PES, but has the major drawback that the equation of motion for the nuclei is non-local in time (i.e., the force on the nuclear DOF at time $t$ involves the future and the past), and is thus very difficult to implement. Were this two-step procedure carried out within the SC-IVR/MMST formulation, the first step - calculation of the electronic transition for a fixed nuclear trajectory — would be done exactly, because the time-dependent 'electronic' Hamiltonian would be a time-dependent quadratic Hamiltonian, for which the SC-IVR is exact. And the second step would be done more accurately and efficiently by the SC-IVR than via the stationary phase approximation. Thus the SC-IVR/MMST approach must be more accurate than that of Pechukas, and more importantly, it is easier to implement since it evolves the electronic and nuclear dynamics simultaneously and thus has no non-local character. 
In summary, the MMST Hamiltonian, Eq. 2.16 or 2.17, provides an extremely useful representation of the nuclear and electronic DOF of a molecular system, and one sees again that all quantum effects in molecular dynamics are described at least qualitatively correctly by a consistent SC treatment of all the DOF of the system, and that the description is usually sufficiently quantitative to be useful. Particularly interesting is that the nuclear-electronic classical trajectories generated by the MMST Hamiltonian (and which go into the SC theory) are 'Ehrenfest trajectories'. Used within the SC framework, though, they correctly describe nuclear motion that 'settles down' on one electronic PES or another after some time in regions where the electronic coupling vanishes. The primary remaining challenges are to develop the methodologies and algorithms necessary to implement this formulation as efficiently as possible.

\section{Acknowledgment}

I would like to thank Prof. Eitan Geva for hosting my sabbatical at the University of Michigan

for the fall semester, 2008, during which time this paper was written. This work was supported by the Director, Office of Science, Office of Basic Energy Sciences, Chemical Sciences, Geosciences, and Biosciences Division, U.S. Department of Energy under Contract No. DEAC02-05CH1 1231, by the National Science Foundation Grant No. CHE-0809073 and by the Office of Naval Research Grant Nos. N00014-07-1-0586 and N00014-05-1-0457.

\section{References}

1. (a) W. H. Miller, J. Chem. Phys. 53, 3578-3587 (1970).

(b) R. A. Marcus, J. Chem. Phys. 54, 3965 (1971).

(c) W. H. Miller, Adv. Chem. Phys. 25, 69 (1974); 30, 77 (1975). 
(d) E. J. Heller, J. Chem. Phys. 62, 1544 (1975).

2. Interestingly, the motivation for using $\mathrm{SC}$ methods now is essentially the same as it was in the 1960's and 70's. The 1960's saw the first use of numerically computed classical trajectories to describe inelastic and reactive collisions of small molecular systems (e.g., A + $\mathrm{BC} \rightarrow \mathrm{AB}+\mathrm{C})$, and they played a very important role since it was not possible at that time to carry out the corresponding fully quantum calculations. Similarly today, one would be happy to solve the Schrödinger equation for large molecular systems if it were possible. Numerically computed classical trajectory calculations are possible, though, and are immensely useful. The SC idea, now and then, is to take these numerically computed classical trajectories and use them as input to an SC description, thereby having all of the classical mechanics correct and an approximation description of the quantum effects.

3. (a) M. F. Herman and E. Kluk, Chem. Phys. 91, 27 (1984).

(b) K. G. Kay, J. Chem. Phys. 100, 4377, 4432 (1994).

(c) G. Campolieti and P. Brumer, Phys. Rev. A 50, 997 (1994).

(d) E. J. Heller, J. Chem. Phys. 94, 2723 (1991).

4. (a) D. J. Tanner and S. Garaschuk, Ann. Rev. Phys. Chem. 51, 553 (2000).

(b) W. H. Miller, J. Phys. Chem. A 105, 2942-2955 (2001).

(c) W. H. Miller, Proceedings of the National Academy of Sciences 102, 6660-6664 (2005).

(d) W. H. Miller, J. Chem. Phys. 125, 132305.1-8 (2006).

(e) K. G. Kay, Ann. Rev. Phys. Chem. 56, 255 (2005).

5. I don't know who first used this approach for treating electronically non-adiabatic processes. It is also sometimes used on one PES to treat a vibrational DOF quantum mechanically, and all the other nuclear DOF classically. An early example is (a) G. D. 
Billing, Chem. Phys. Lett. 30, 391 (1975), and a very recent one is (b) A. Bastida, J. Zuniga, A. Requena, and B. Miquiel, J. Chem. Phys. 129, 154501 (2008).

6. One can equally well use the adiabatic representation for the $\mathrm{N}$ electronic states - see Eq. 2.17 below - but it is simpler to discuss the development using a diabatic representation.

7. J. C. Tully and R. K. Preston, J. Chem. Phys. 55, 562 (1971).

8. N. Ananth, C. Venkataraman and W. H. Miller, J. Chem. Phys. 127, 084114.1-9 (2007).

9. J. C. Tully, J. Chem. Phys. 60, 3042 (1974).

10. F. Rebentrost and W. A. Lester, J. Chem. Phys. 67, 3367 (1977).

11. K. Bergmann, S. R. Leone, and C. B. Moore, J. Chem. Phys. 63, 4161 (1975).

12. D. J. Nesbit and S. R. Leone, J. Chem. Phys. 73, 6182 (1980).

13. W. H. Miller, J. Chem. Phys. 68, 4431-4434 (1978).

14. (a) L. D. Landau, Physik. Z. Sowjetunion URSS 2, 46 (1932).

(b) C. Zener, Proc. Roy. Soc. (London) A 137, 696 (1932).

(c) E. C. G. Stuckelberg, Helv. Phys. Acta 5, 369 (1932).

15. W. H. Miller and T. F. George, J. Chem. Phys. 56, 5637-5652 (1972).

16. W. H. Miller and C. W. McCurdy, J. Chem. Phys. 69, 5163-5173 (1978).

17. C. W. McCurdy, H. D. Meyer, and W. H. Miller, J. Chem. Phys. 70, 3177-3187 (1979).

18. H. D. Meyer and W. H. Miller, J. Chem. Phys. 70, 3214-3223 (1979).

19. P. A. M. Dirac, Proc Roy. Soc. (London) A114, 243 (1927).

20. See, for example, L. M. Raff and D. L. Thompson, in The Theory of Reaction Dynamics, ed. M. Baer, CRC Press, 1985, Vol. 3, p.1.

21. See, for example, R. N. Porter, L. M. Raff, and W. H. Miller, J. Chem. Phys. 63, 2214-2218 (1975). 
22. H. D. Meyer and W. H. Miller, J. Chem. Phys. 71, 2156-2169 (1979).

23. D. P. Ali and W. H. Miller, Chem. Phys. Lett. 103, 470-474 (1984).

24. S. K. Gray and W. H. Miller, Chem. Phys. Lett. 93, 341-344 (1982).

25. M. B. Faist and R. D. Levine, J. Chem. Phys. 64, 2953 (1976).

26. G. Stock and M. Thoss, Phys. Rev. Lett. 78, 578 (1997).

27. X. Sun and W. H. Miller, J. Chem. Phys. 108, 8870-8877 (1998).

28. (a) B. B. Harland and P. N. Roy, J. Chem. Phys. 118, 4791 (2003); (b) B. B. Issack and P.N. Roy, J. Chem. Phys. 127, 144306 (2007).

29. (a) H. Wang, X. Sun, and W. H. Miller, J. Chem. Phys. 108, 9726-9736 (1998).

(b) X. Sun, H. Wang, and W. H. Miller, J. Chem. Phys. 109, 4190-4200 (1998).

30. For example,

(a) K. Imne, E. Ozizmir, M. Rosenbaum and P. F. Zweifel, J. Math Phys. 810907 (1967).

(b) W. H. Miller, J. Chem. Phys. 61, 1823-1834 (1974).

(c) E. J. Heller, J. Chem. Phys. 65, 1289 (1976).

(d) H. W. Lee and M. O. Scully, J. Chem. Phys. 73, 2238 (1980).

(e) J. L. Liao and E. Pollak, J. Chem. Phys. 108, 2733 (1998).

(f) J. A. Poulsen, G. Nyman and P. J. Rossky, J. Chem. Phys. 119, 12179 (2003).

31. The two trajectories in Eq. 3.6 can both be considered to start at $t=0$, with the IVR expression of one being complex-conjugated, or equivalently one can think of one propagating forward in time from 0 to $t$, and the other propagating backward in time from $t$ to 0 .

32. Y. Zhao and W. H. Miller, J. Chem. Phys. 117, 9605-9610 (2002).

33. J. Liu and W. H. Miller, J. Chem. Phys. 127, 114506.1-10 (2007). 
34. (a) W. H. Miller, Faraday Disc. Chem. Soc. 110, 1-21 (1998).

(b) X. Sun and W. H. Miller, J. Chem. Phys. 110, 6635-6644 (1999).

35. N. Makri and K. Thompson, Chem. Phys. Lett. 291, 101 (1998).

36. X. Sun and W. H. Miller, J. Chem. Phys. 106, 6346-6353 (1997).

37. (a) V. S. Batista and W. H. Miller, J. Chem. Phys. 108, 498-510 (1998).

(b) X. Sun, H. Wang, and W. H. Miller, J. Chem. Phys. 109, 7064-7074 (1998).

(c) H. Wang, X. Song, D. Chandler and W. H. Miller, J. Chem. Phys. 110, 4828-4840 (1999).

(d) M. Thoss and G. Stock, Phys. Rev. A 59, 64 (1999).

(e) E. Rabani, S. A. Egorov, B. J. Berne, J. Phys. Chem. A 103, 9539 (1999).

(f) M. Thoss, W. H. Miller and G. Stock, J. Chem. Phys. 112, 10282-10292 (2000).

(g) S. Bonella and D. F. Coker, J. Chem. Phys. 114, 7778 (2001).

(h) S. Dilthey and G. Stock, Phys. Rev. Lett. 87, 140404 (2001).

(i) J.-L. Liao and G. A. Voth, J. Phys. Chem. B 106, 8449 (2002).

(j) D. V. Shalashilin and M. S. Child, J. Chem. Phys. 121, 3563 (2004).

(k) A. Novikov, U. Kleinkathoefer and M. Schreiber, Chem. Phys. 296, 149 (2004).

(1) Q. Shi and E. Geva, J. Phys. Chem. A 108, 6109 (2004).

(m) H. Kim, A. Nassimi and R. Kapral, J. Chem. Phys. 129, 084102 (2008).

38. G. Stock, M. Thoss, Adv. Chem. Phys. 134243 (2005).

39. F. Grossmann, “Theoretical Femtosecond Physics”, Springer, Heidelberg (2008).

40. J. C. Tully, J. Chem. Phys. 93, 1061 (1990).

41. K. F. Wong and P. J. Rossky, J. Chem. Phys. 116, 8418 (2002).

42. C. Zhu, A. W. Jasper, D. G. Truhlar, J. Chem. Phys. 120, 5543 (2004). 
43. X. Sun and W. H. Miller, J. Chem. Phys. 106, 916-927 (1997).

44. P. Pechukas, Phys. Rev 181, 174 (1969).

\section{Figure Captions}

Figure 1. Sketch of the adiabatic potential difference $\Delta \mathrm{W}(\mathrm{t})$ along a classical trajectory, with local minima that correspond to 'avoided crossings'; cf. the discussion in Sec IIa following Eq 2.1. (a) is characteristic of a very slow collision, with many vibrational periods that lead to many local minima, spaced by the vibrational period; (b) is characteristic of a higher energy collision with fewer vibrational periods during the interaction time, and thus fewer local minima. (From ref. 13)

Figure 2. Cross section for quenching of $\mathrm{F}^{*}\left({ }^{2} \mathrm{P}_{1 / 2}\right)$ by collision with $\mathrm{H}_{2}(\mathrm{j}=0)$, as a function of initial translational energy E. The solid curves are the quantum mechanical results of ref. 22, and the points the results of the 'quasi-classical' trajectory calculations described in Sec IIb. Note the break in the scale; i.e., the cross section for rotationally excited $\mathrm{H}_{2}(\mathrm{j}=2)$ is more than an order of magnitude larger. (From ref. 22)

Figure 3. Cross section for quenching of $\mathrm{Br}^{*}\left({ }^{2} \mathrm{P}_{1 / 2}\right)$ by collision with $\mathrm{H}_{2}(\mathrm{v}=0)$, as a function of initial translational energy, given by quasi-classical trajectory calculations as discussed in Sec IIb. The crosses and solid circles are for final $\mathrm{H}_{2}$ vibrational state $\mathrm{v}=1$ and 0 , respectively; i.e., the cross section for vibrationally excited $\mathrm{H}_{2}$ is much larger. (The error bars denote the usual Monte Carlo error estimate.) (From ref. 23)

Figure 4. Cross section for the charge transfer process $\mathrm{Na}+\mathrm{I} \rightarrow \mathrm{Na}^{+}+\mathrm{I}^{-}$, as a function of initial translational energy. The solid curve is the quantum coupled channel calculation from ref. 24, and the open circles those of the quasi-classical trajectory calculations discussed in Sec IIb. 
(The dashed curve is the results of a perturbative approximation not related to the present discussion.) (From ref. 24)

Figure 5. Potential curves for the (a) symmetric and (b) asymmetric versions of the electronically non-adiabatic scattering problem discussed in Sec IIIc. Both diabatic and adiabatic potentials are shown. The two arrows on the vertical (energy) axes indicate the two collision energies for which calculations were carried out. Note that for the asymmetric version (Fig 5b), at the lower energy the higher electronic state is a closed channel (i.e., energetically forbidden) in the 'product' region (transmission) $\mathrm{R}>>0$, while both states are allowed for reflection, $\mathrm{R}<<0$.

Figure 6. Probability distribution of the final $(\mathrm{t} \rightarrow \infty)$ translational momentum, as defined by Eq. 3.9, for the symmetric potential (Fig. 5a) and the higher energy. (a) Solid line is the numerically exact QM result, and dashed line that given by the FB-IVR. (b) Solid line (which is a schematic depiction of a Dirac delta function) is the result given by the traditional Ehrenfest model, and dashed line that given by the classical Wigner model (or linearized SC-IVR = LSCIVR) approximation.

Figure 7. Same as Fig. 6, except for the lower energy indicated in Fig. 5a.

Figure 8. Same as Fig. 6, except for the asymmetric potentials of Fig. 5b (for the higher energy indicated).

Figure 9. Same as Fig. 8, except for the lower energy indicated in Fig. 5b. 

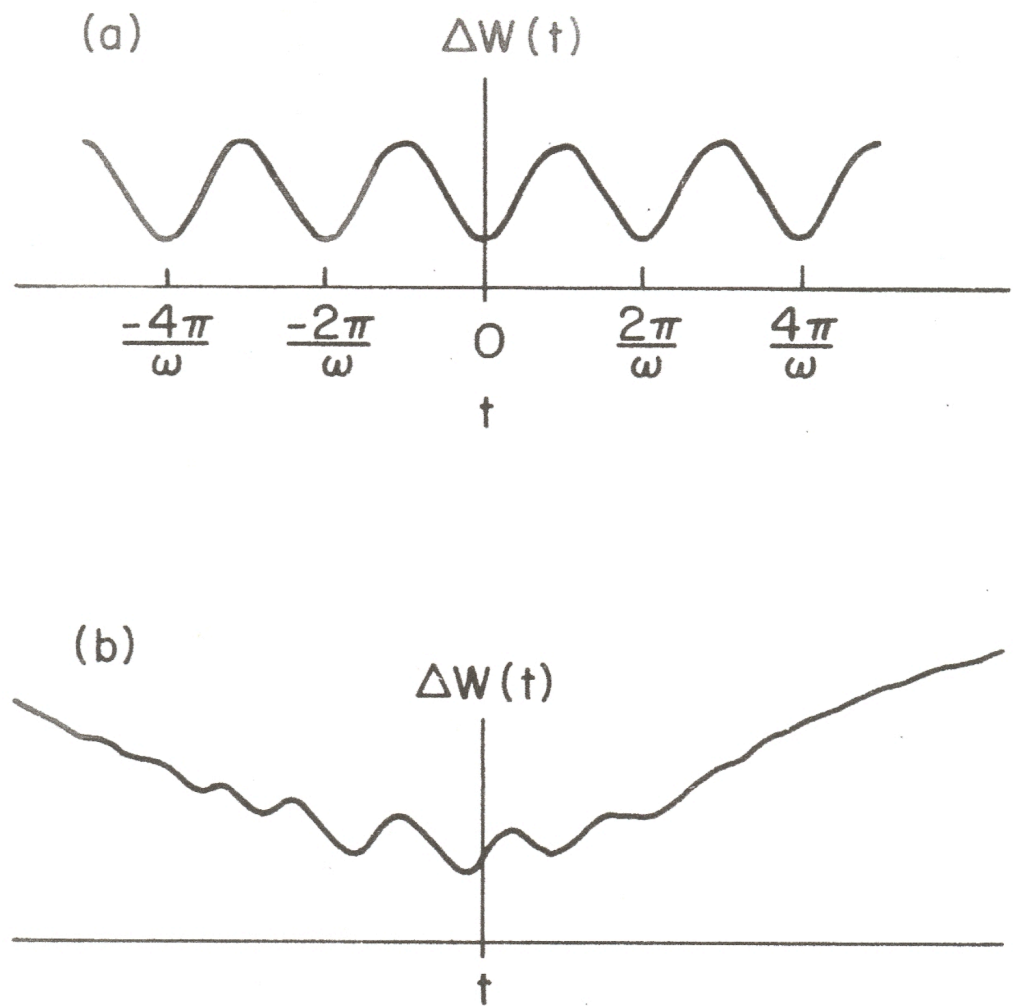

Figure 1 


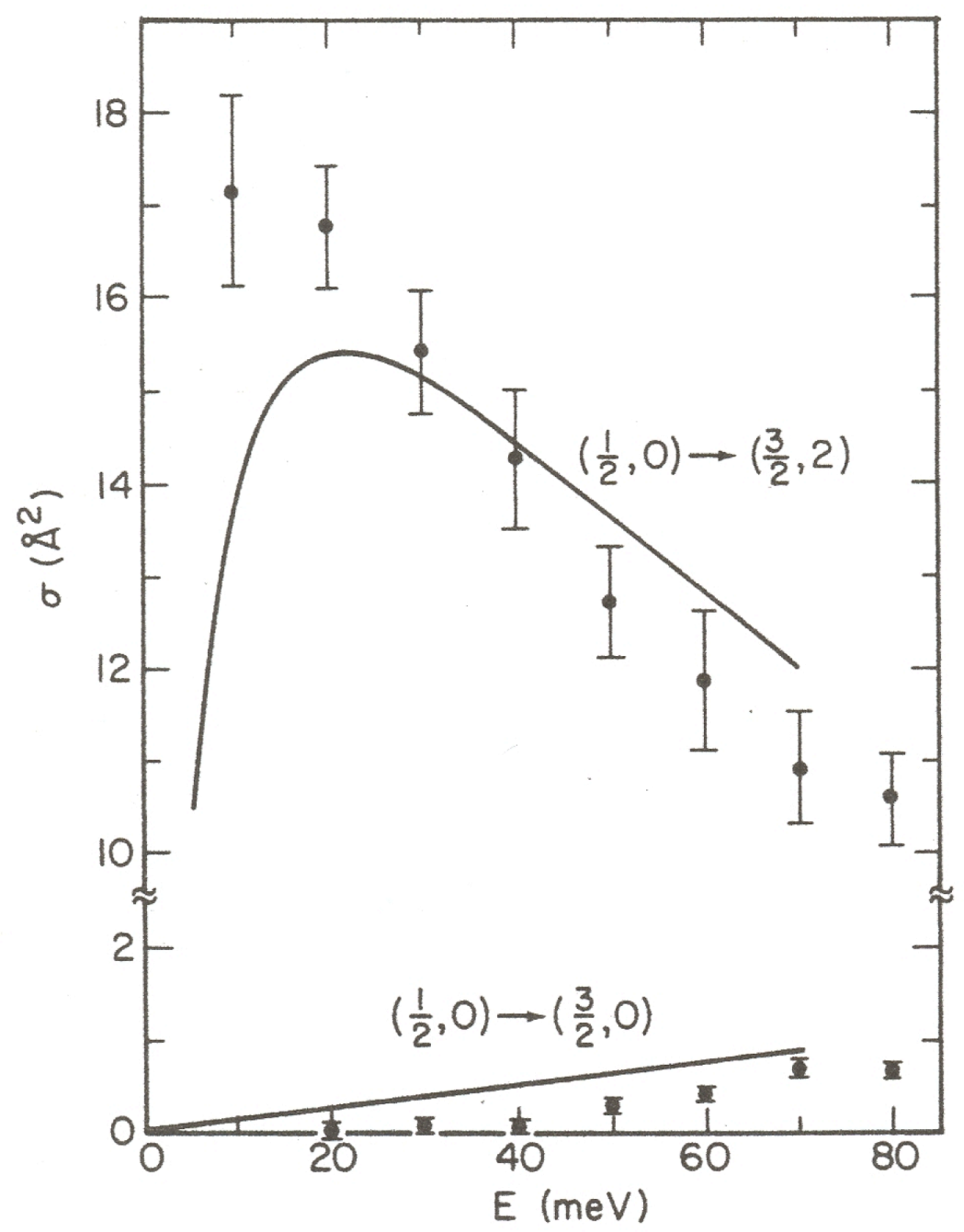

Figure 2 


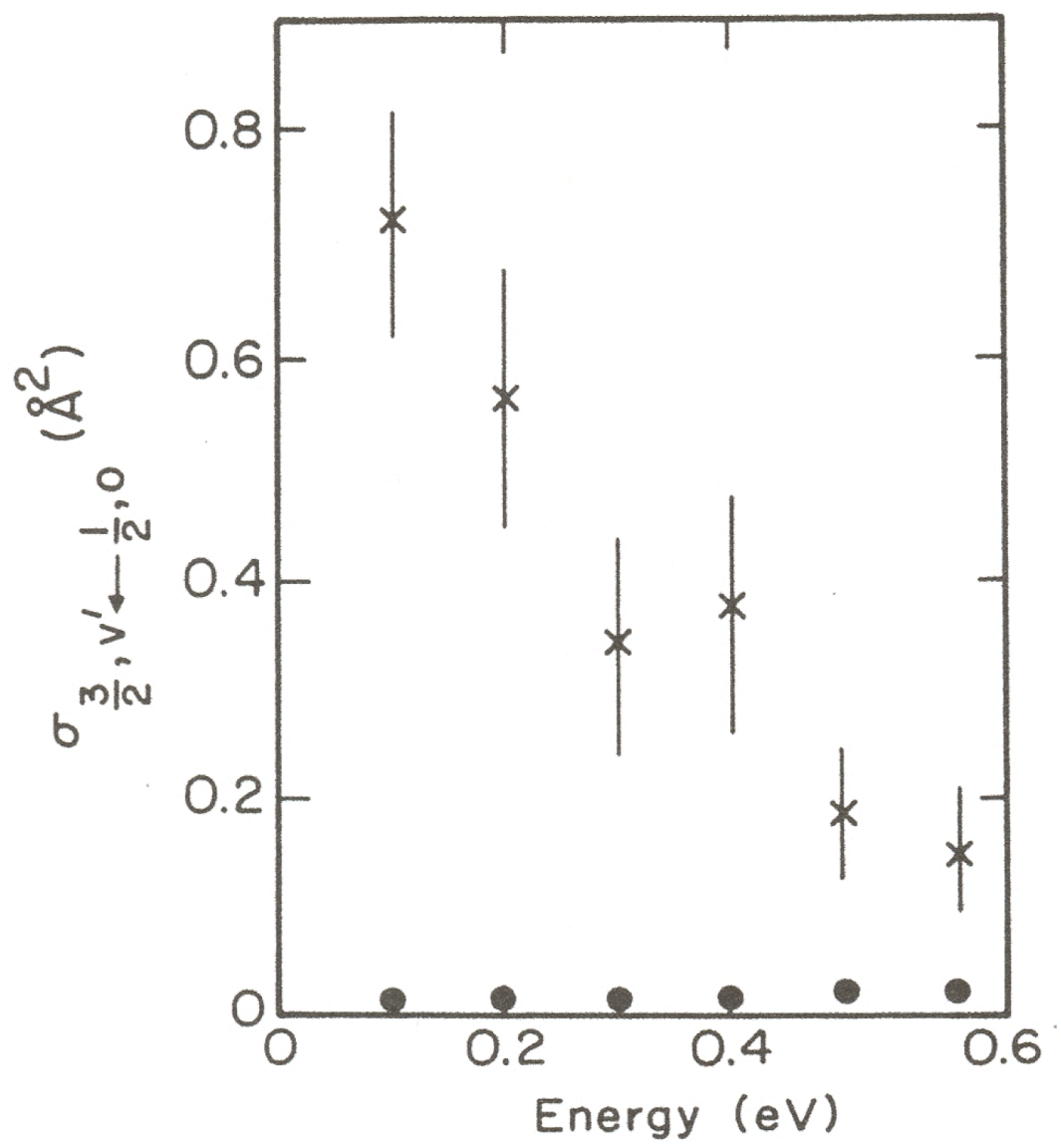

Figure 3 


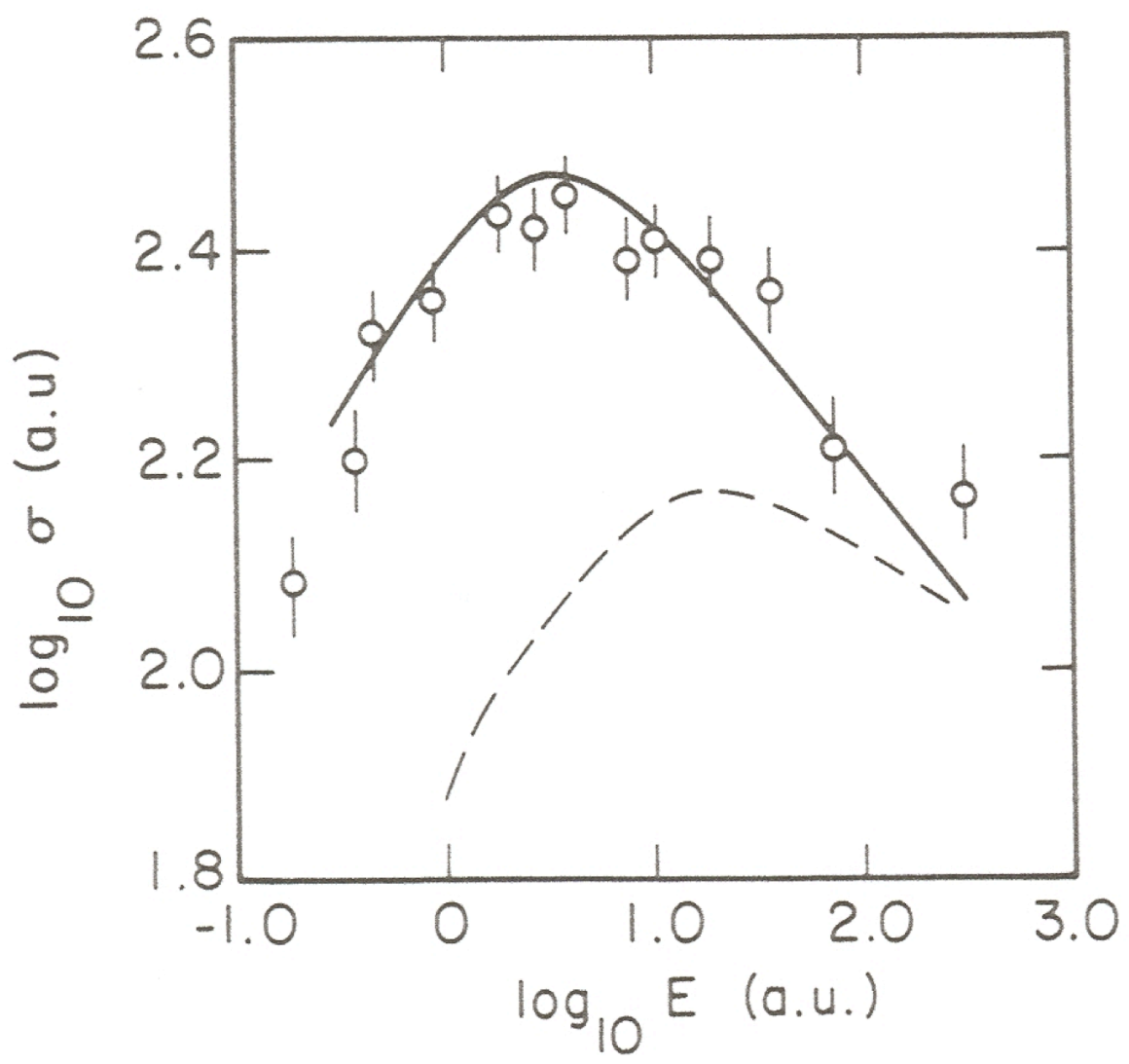

Figure 4 


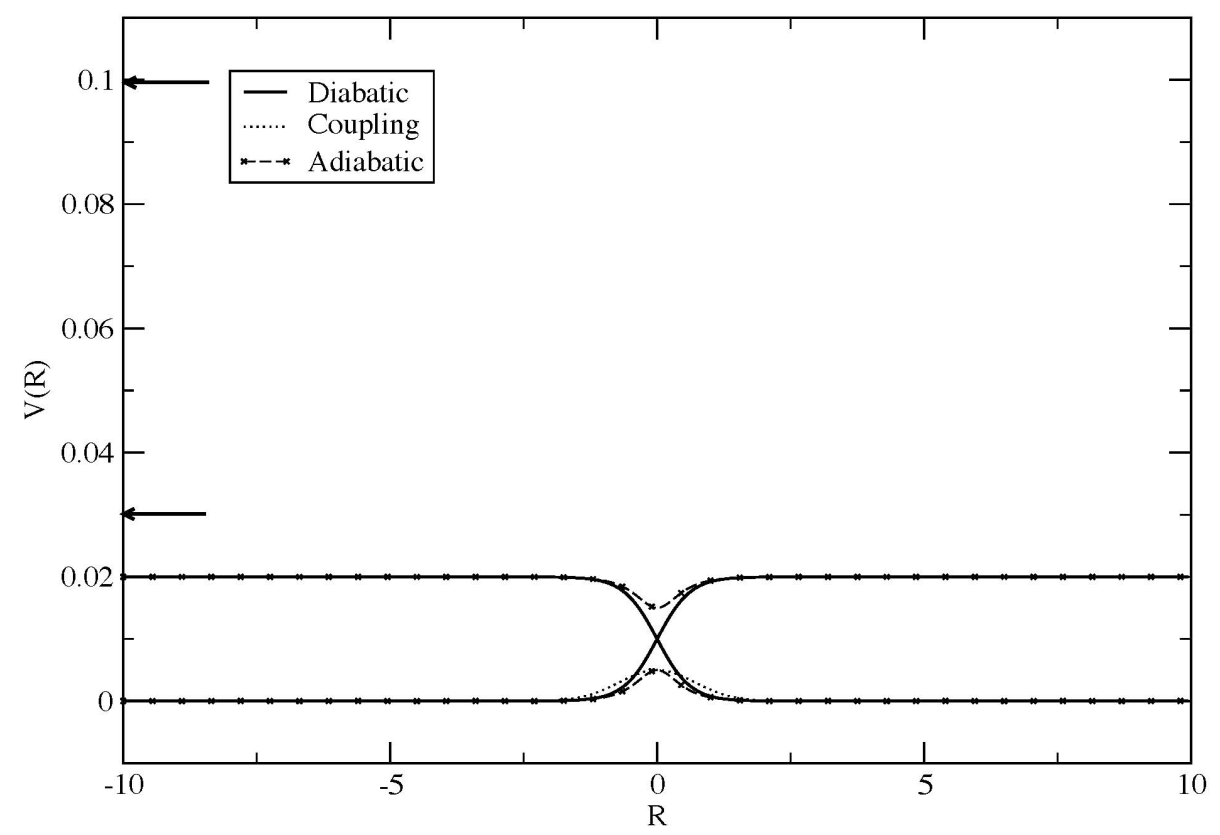

Figure 5a

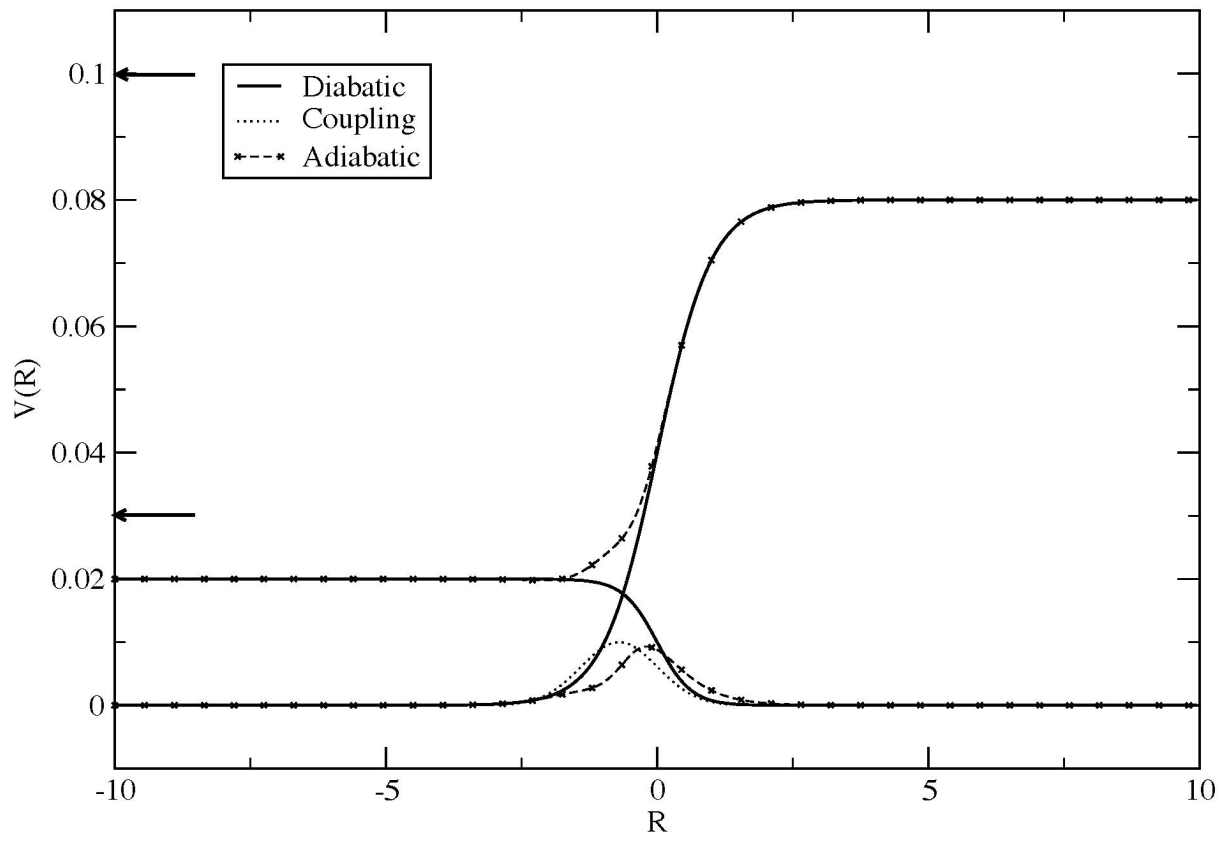

Figure 5b 


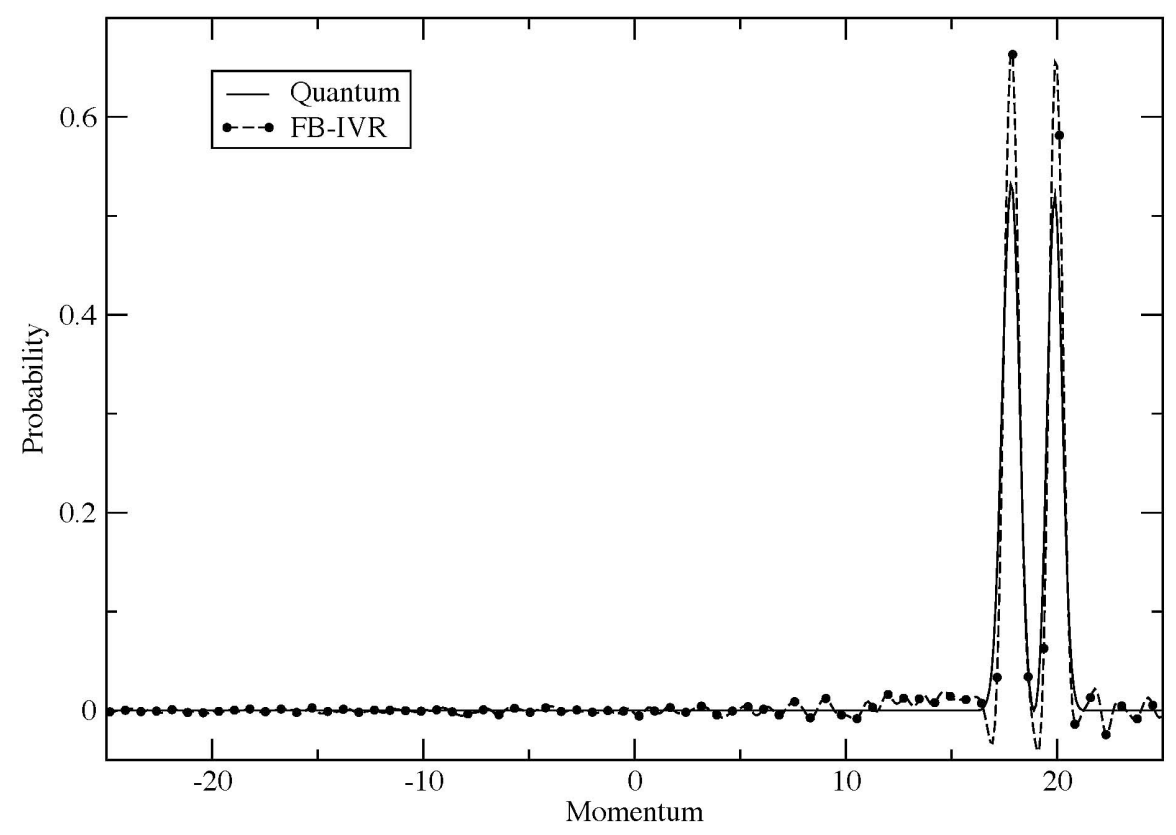

Figure 6a

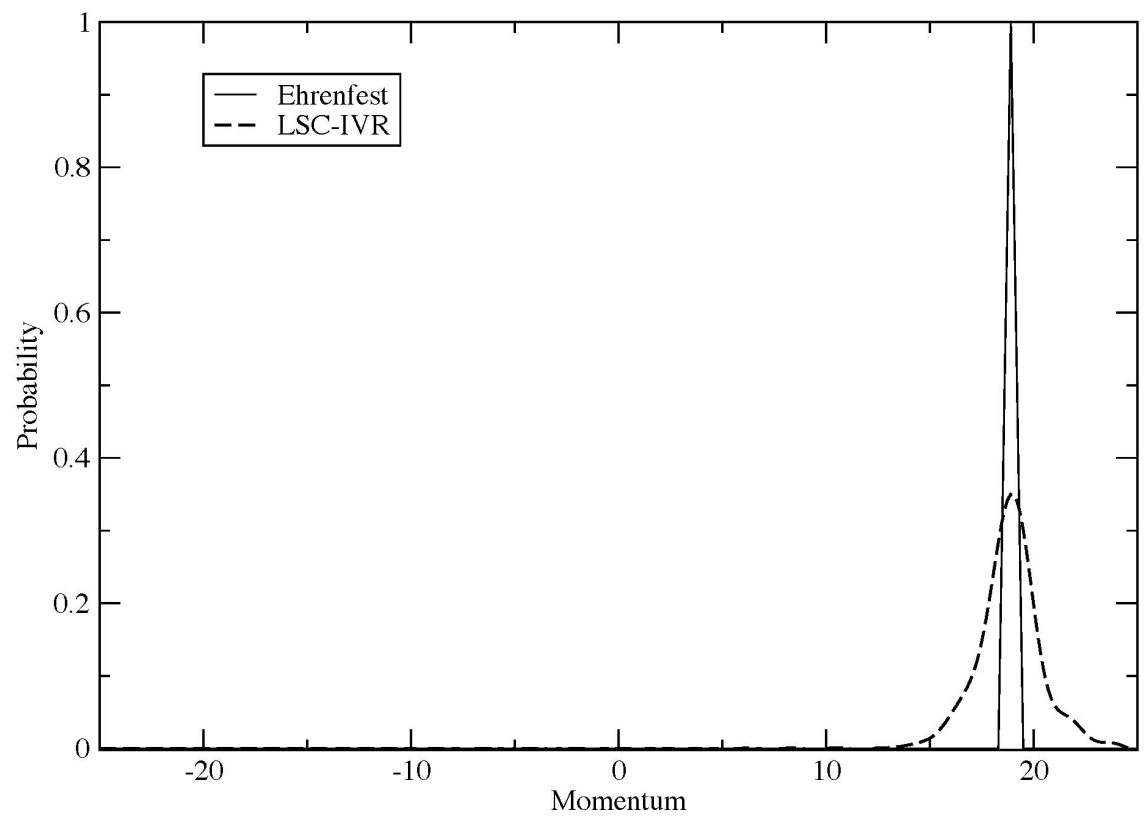

Figure $6 b$ 


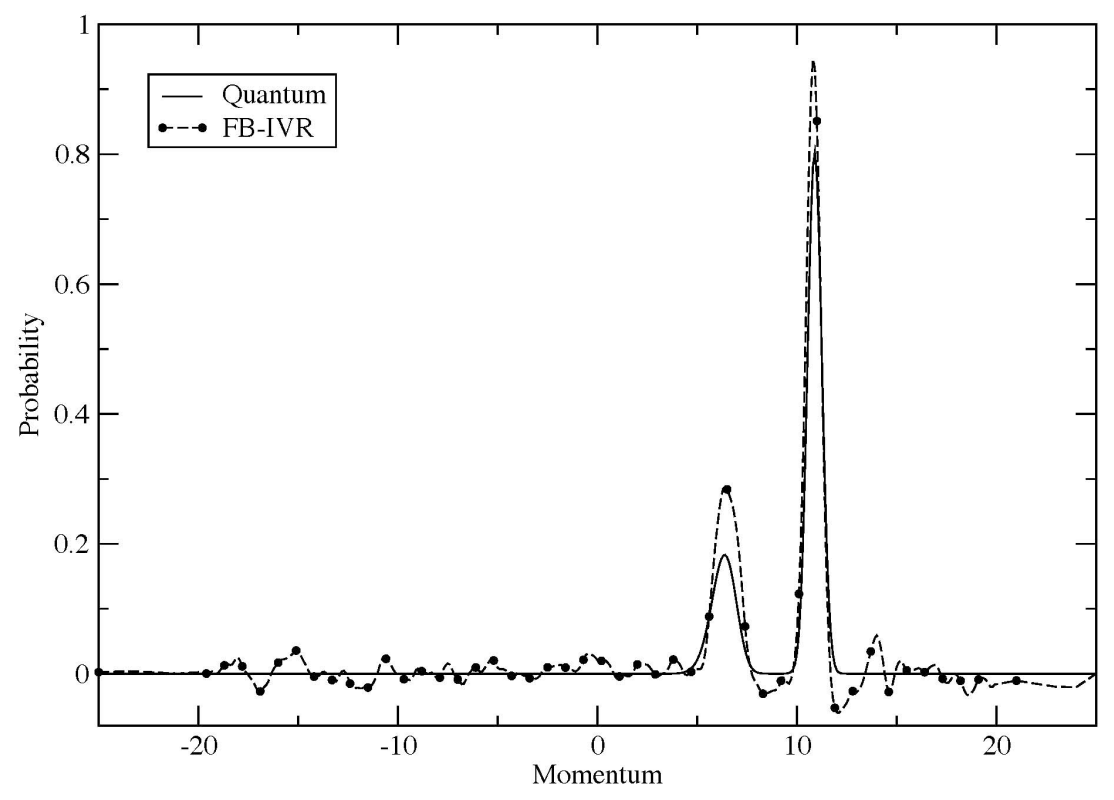

Figure $7 \mathrm{a}$

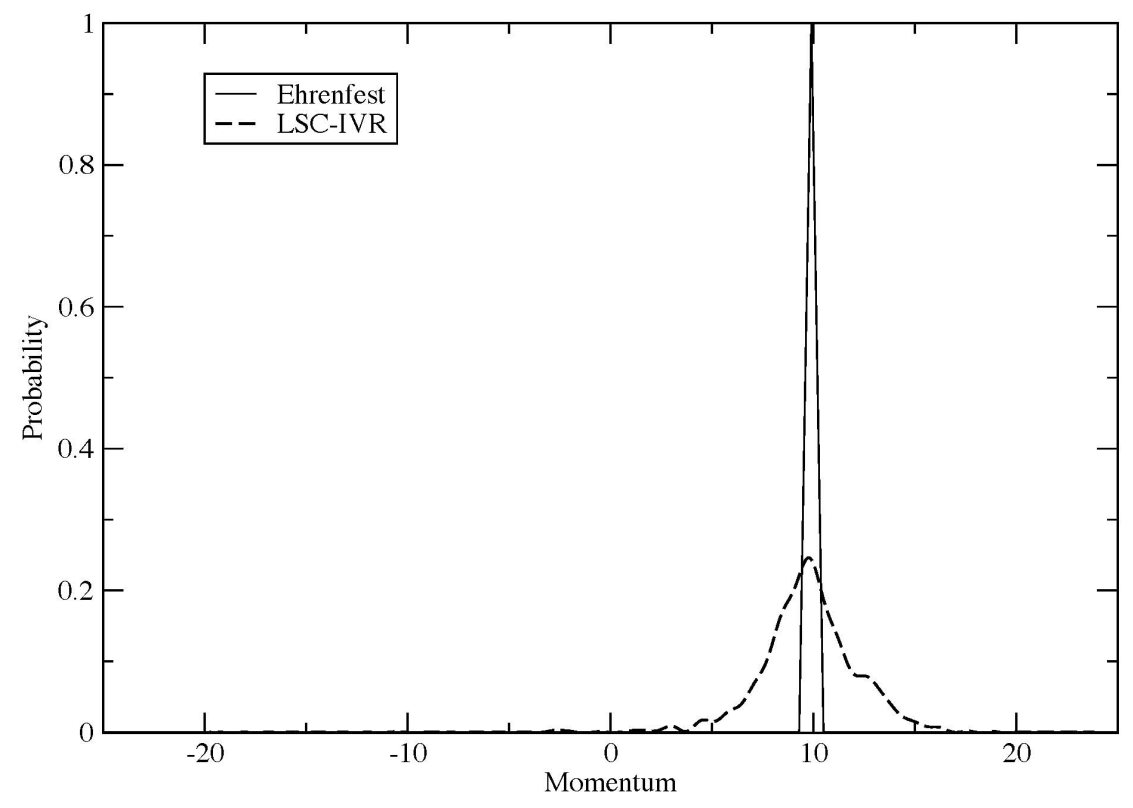

Figure $7 b$ 


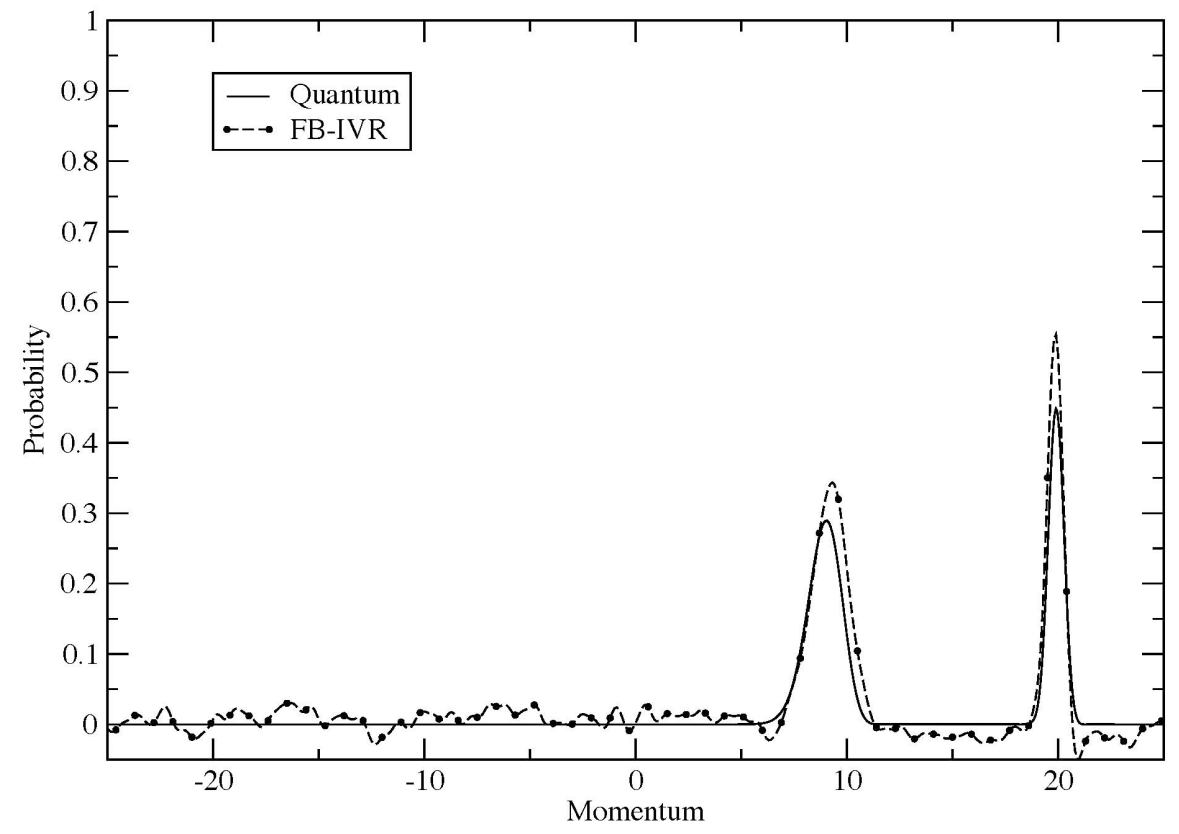

Figure 8a

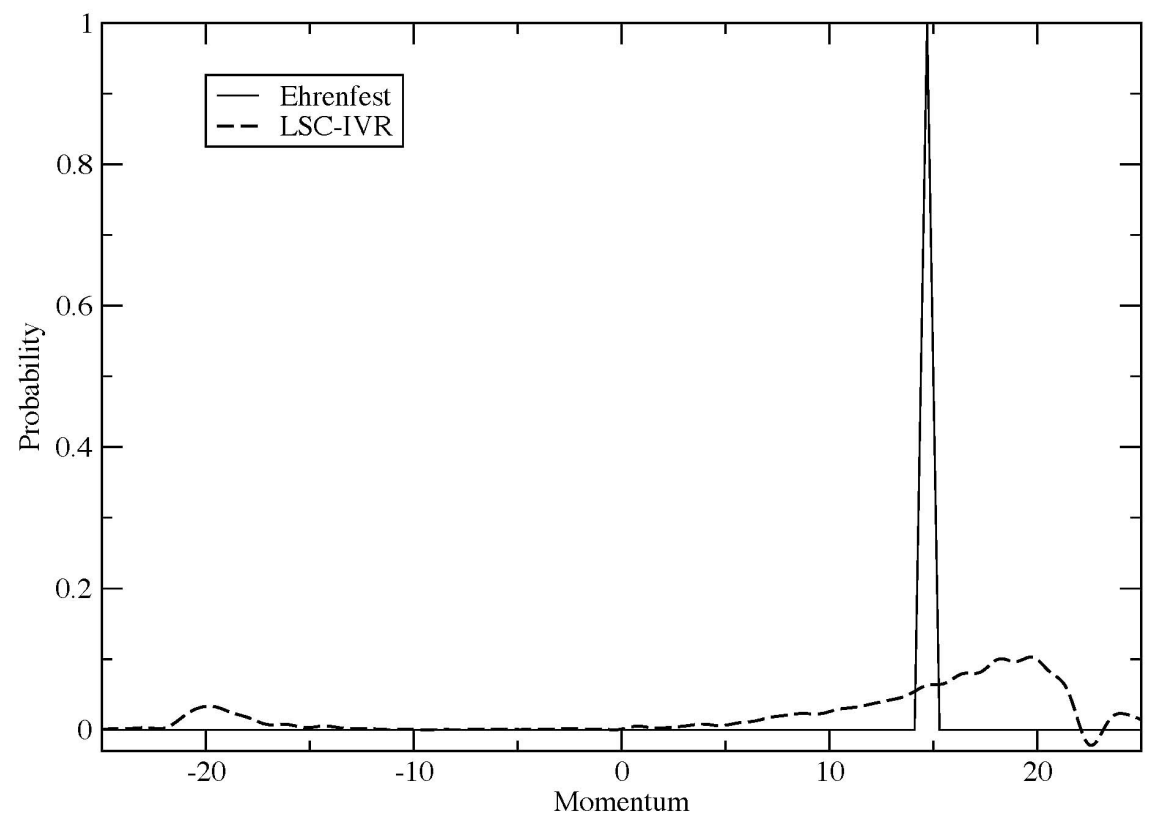

Figure $8 b$ 


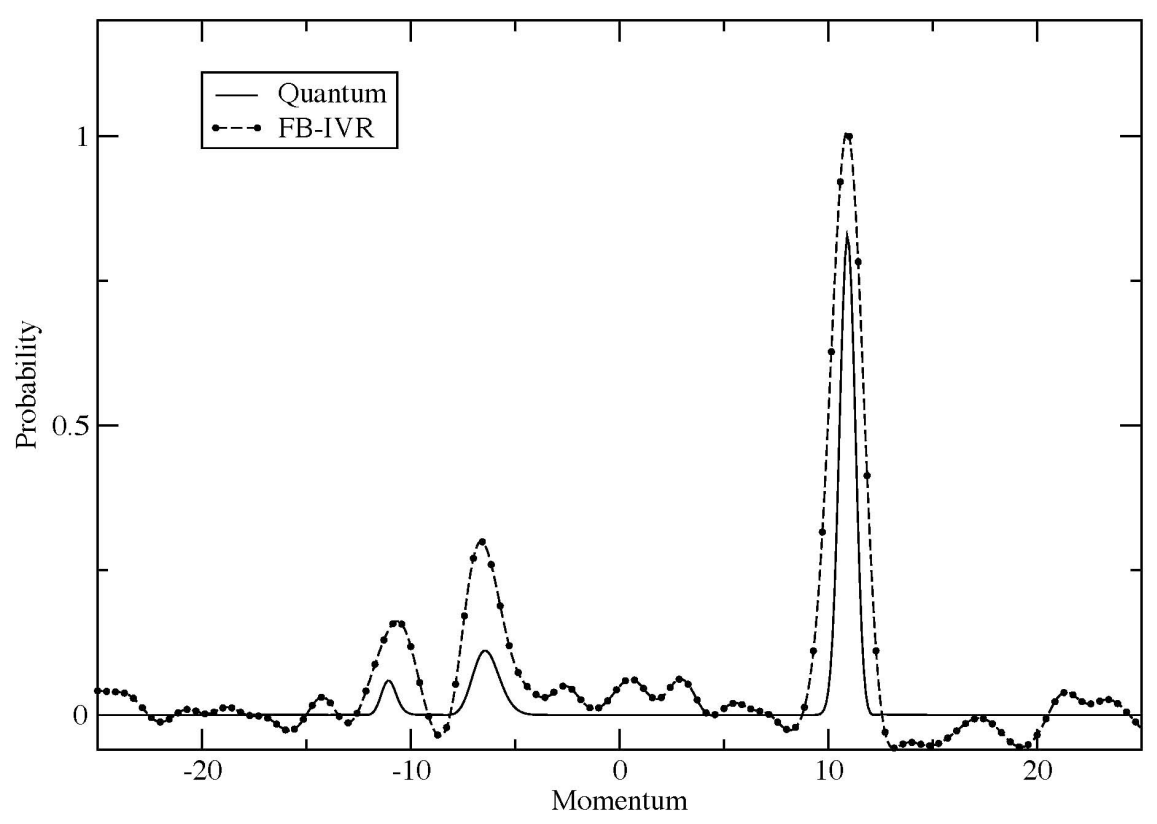

Figure 9a

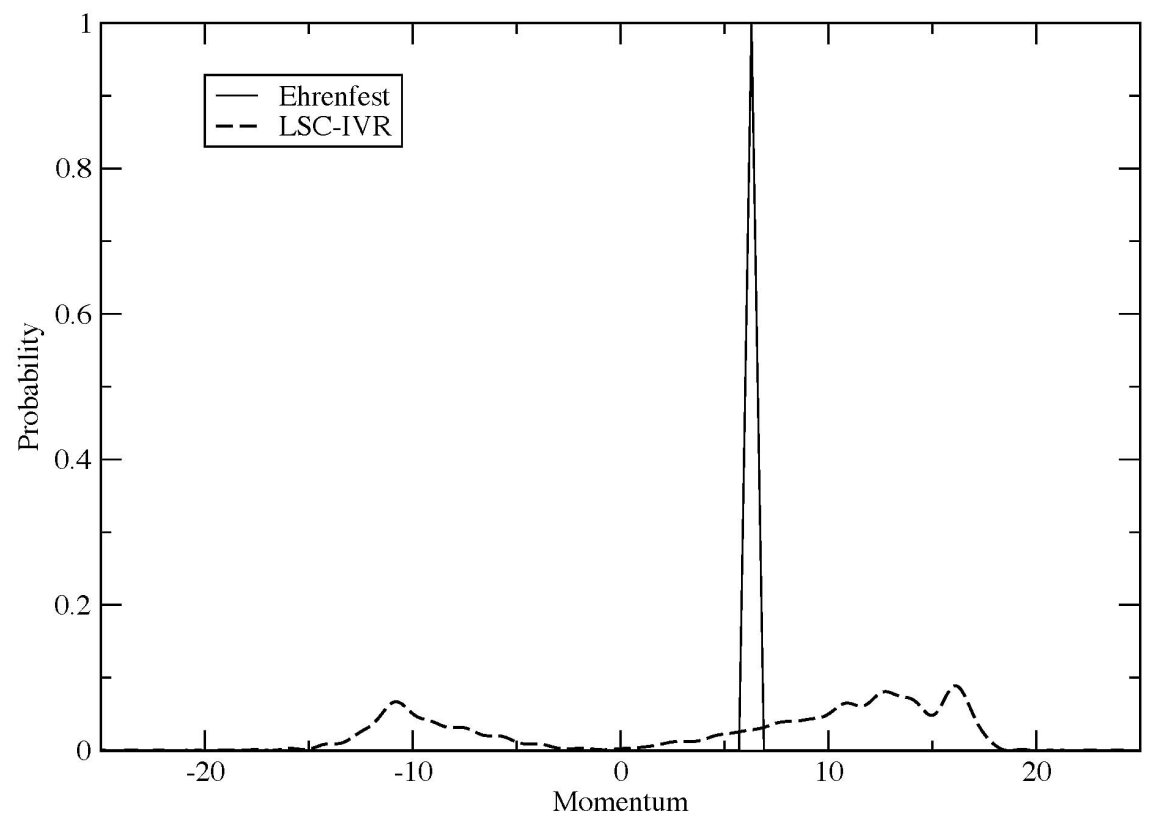

Figure 9b 